\title{
Telescope Array Radar (TARA) Observatory for Ultra-High Energy Cosmic Rays
}

\author{
R. Abbasia ${ }^{\mathrm{a}}$, M. Abou Bakr Othman ${ }^{\mathrm{a}}$, C. Allen ${ }^{\mathrm{b}}$, L. Beard ${ }^{\mathrm{c}}$, J. Belz ${ }^{\mathrm{a}}$, D. Besson ${ }^{\mathrm{b}, \mathrm{g}}$, M. Byrne $^{\mathrm{a}}$, \\ B. Farhang-Boroujeny ${ }^{\mathrm{a}}$, A. Gardner ${ }^{\mathrm{a}}$, W.H. Gillman ${ }^{\mathrm{d}}$, W. Hanlon ${ }^{\mathrm{a}}$, J. Hanson ${ }^{\mathrm{b}}$, C. Jayanthmurthy ${ }^{\mathrm{a}}$, S. Kunwar ${ }^{\mathrm{b}}$, \\ S.L. Larson ${ }^{\mathrm{e}}$, I. Myers ${ }^{\mathrm{a}, *}$, S. Prohira ${ }^{\mathrm{b}}$, K. Ratzlaff ${ }^{\mathrm{b}}$, P. Sokolsky ${ }^{\mathrm{a}}$, H. Takai ${ }^{\mathrm{f}}$, G.B. Thomson ${ }^{\mathrm{a}}$, D. Von Maluski ${ }^{\mathrm{a}}$ \\ ${ }^{a}$ University of Utah, Salt Lake City, UT 84112 U.S.A. \\ ${ }^{b}$ University of Kansas, Lawrence, KS 66045 U.S.A. \\ ${ }^{c}$ Purdue University, West Lafayette, IN 47907 U.S.A. \\ ${ }^{d}$ Gillman $\mathcal{E}$ Associates, Salt Lake City, UT 84106 U.S.A. \\ ${ }^{e}$ Utah State University, Logan, Utah 84322 U.S.A. \\ ${ }_{\text {B }}$ rookhaven National Laboratory, Upton, NY 11973 U.S.A. \\ ${ }^{g}$ Moscow Engineering and Physics Institute, 31 Kashirskaya Shosse, Moscow 115409 Russia
}

\begin{abstract}
Construction was completed during summer 2013 on the Telescope Array RAdar (TARA) bi-static radar observatory for Ultra-High-Energy Cosmic Rays (UHECR). TARA is co-located with the Telescope Array, the largest "conventional" cosmic ray detector in the Northern Hemisphere, in radio-quiet Western Utah. TARA employs an 8 MW Effective Radiated Power (ERP) VHF transmitter and smart receiver system based on a $250 \mathrm{MS} / \mathrm{s}$ data acquisition system in an effort to detect the scatter of sounding radiation by UHECR-induced atmospheric ionization. TARA seeks to demonstrate bi-static radar as a useful new remote sensing technique for UHECRs. In this report, we describe the design and performance of the TARA transmitter and receiver systems.
\end{abstract}

Keywords: cosmic ray, FPGA, radar, digital signal processing, chirp

\section{Introduction}

Cosmic rays with energies per nucleon in excess of $\approx 10^{14} \mathrm{eV}$ [1] create cascades of particles with electromagnetic and hadronic components in the atmosphere, known as Extensive Air Showers (EAS). Conventional cosmic ray experiments detect events through coincident shower front particles in an array of surface detectors [2, 3] or through fluorescence photons that radiate from the shower core [4, 5, 6] which permit fluorescence telescopes to study shower longitudinal development. Another technique takes advantage of two naturally-emitted radio signals: the Askaryan effect [7] and geomagnetic radiation from interactions with the Earth's magnetic field [8].

With ground arrays, air shower particles are observed directly. The land required to instrument ground arrays is large, $c f$. Telescope Array's $700 \mathrm{~km}^{2}$ surface detector covers roughly the same land area as New York City. The costs of the equipment required to instrument such

${ }^{*}$ Corresponding Author. Tel.: +01 801 5879986. Addr.: $115 \mathrm{~S}$ 1400 E \#201 JFB

Email address: isaac@cosmic .utah.edu (I. Myers) a large area are substantial, and the available land can only be found in fairly remote areas.

A partial solution to the difficulties and expense involved in ground arrays is found in the fluorescence technique. Here, the atmosphere itself is part of the detection system, and air shower properties may be determined at distances as remote as $40 \mathrm{~km}$. Unfortunately fluorescence observatories are typically limited to a ten percent duty cycle by the sun, moon and weather.

The possibility of radar observation of cosmic rays dates to the 1940's, when Blackett and Lovell [9] proposed cosmic rays as an explanation of anomalies observed in atmospheric radar data. At that time, a radar facility was built at Jodrell Bank to detect cosmic rays, but no results were ever reported. Recent experimental efforts utilizing atmospheric radar systems were conducted at Jicamarca [10] and at the MU-Radar [11]. Both observed a few signals of short duration indicating a relativistic target. However in neither case were the measurements made synchronously with a conventional cosmic ray detector.

A new approach, first attempted by the MARIACHI [12, 13] project, is to utilize bi-static or two- 


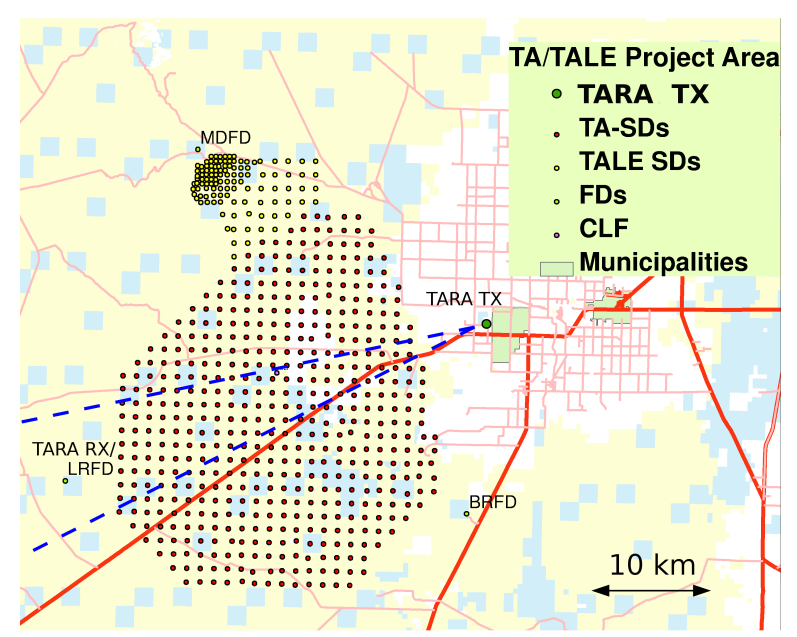

Figure 1: Map of TARA Observatory sites (transmitter and receiver) along with the Telescope Array (TA) detector facilities. The transmitter broadcasts as station WF2XZZ near Hinckley, Utah, towards a receiver site located at the TA Long Ridge Fluorescence Detector (FD). The sounding radiation illuminates the air over the central portion of the TA Surface Detector array, shown with dashed blue lines that indicate the beamwidth $3 \mathrm{~dB}$ below the peak gain.

station radar in conjunction with a conventional set of cosmic ray detectors. Air shower particles move very close to the speed of light, so the Doppler shift is large compared with airplanes or meteors. The bi-static configuration in which the sounding (interrogating) wave Poynting vector is generally perpendicular to shower velocity (as shown in Figure 3) minimizes the large Doppler shift in frequency expected of the reflected signal (see [14, 15], and Section 2 below.) This scenario is unlike that explored in [15] in which the two vectors are roughly anti-parallel. In the latter case, the relativistic frequency shift is maximized. Also, depending on the size of the radar cross section relative to the square of the sounding wavelength, scattering in the forward direction might be enhanced relative to back scatter [16], thus providing an advantage in detecting the faintest echoes in comparison to mono-static radar (ranging radar).

Co-location with a conventional detector allows for definitive coincidence studies to be performed. If coincidences are detected, the conventional detector's information on the shower geometry will allow direct comparison of echo signals with the predictions of air shower Radio Frequency (RF) scattering models.

The Telescope Array Radar (TARA) project is the next logical step in the development of the bi-static radar technique. Whereas MARIACHI made parasitic use of commercial television carriers as a source of sounding radiation (now impossible due to the transition to digital broadcasts), TARA employs a single transmitter in a vacant VHF band which is under the experimentalists' control. The TARA receiver consists of broadband log-periodic antennas, which are read out using a $250 \mathrm{MS} / \mathrm{s}$ digitizer. TARA is co-located with the Telescope Array, a state-of-the-art "conventional" cosmic ray detector, which happens to be located in a low-noise environment. The layout of the TA and TARA detection facilities are shown in Figure 1 .

This work begins with a brief description of the nature of air shower echoes expected for the TARA configuration. Next, we describe the transmitter and receiver system in some detail, including tests of system performance. Finally we describe upgrades to the system which are currently in progress.

\section{Extensive Air Showers, Radar Echoes}

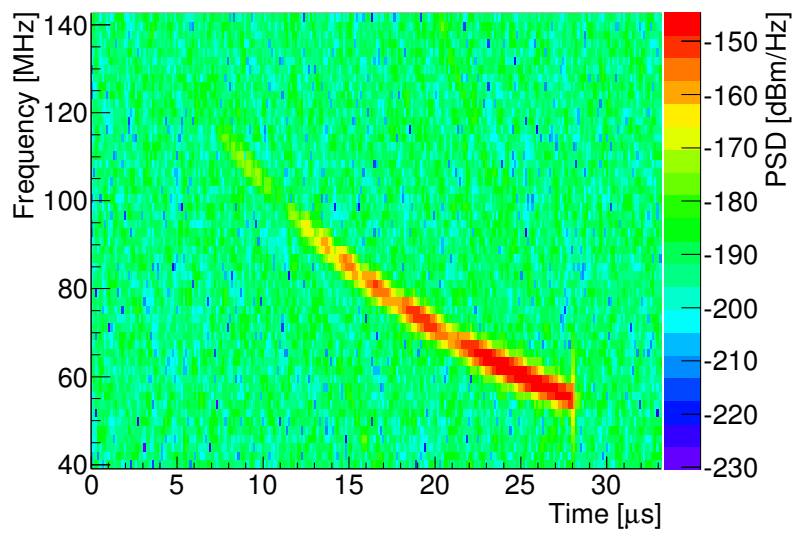

Figure 2: Spectrogram of a chirp signal produced by the radar echo simulation for an EAS located midway between the transmitter and receiver with a zenith angle of $30^{\circ}$ out of the transmitter-receiver plane. A weighted fit to the power of this signal gives a $-2.3 \mathrm{MHz} / \mu \mathrm{s}$ chirp rate. Color scale is Power Spectral Density (PSD) given as $\mathrm{dBm} / \mathrm{Hz}$.

As the EAS core ionizes the atmosphere, liberated charges form a plasma with plasma frequency $v_{p}=$ $(2 \pi)^{-1} \sqrt{n_{e} e^{2} / m_{e} \epsilon_{0}}$, where $n_{e}$ is the electron number density, $e$ is the charge of the electron, and $m_{e}$ is the electron mass. A shower is denoted under-dense or over-dense (See Figure 3 in [17]) relative to the sounding frequency $v$ depending on whether $n_{e}$ corresponds to $v_{p}>v$ or $v_{p}<v$. The radar cross-section of the underdense region is expected to be greatly attenuated due to collisional damping [18, 19, 20]. Therefore, we expect the dominant contribution to EAS radar cross-section $\sigma_{E A S}$ to be the over-dense region, which is modeled as 
a thin-wire conductor [21]. Figure 2] displays a "typical" EAS echo from simulation, where standard shower models of particle production and energy transport have been assumed [22].

The mechanism of radar echo detection of EAS differs from other radio applications because the target is small (i.e., small RCS) and moving near the speed of light. However, letting $R_{T}$ and $R_{R}$ represent the transmitter/shower and receiver/shower distances, respectively, the bi-static geometry (Figure 3 minimizes the phase shift because the total path length $L=R_{R}+R_{T}$ evolves slowly with time. The time-dependence of the path length causes the phase of the echo to evolve, while the transmission maintains a constant frequency. The result is an echo that has a time-dependent frequency a chirp signal [14] (Figure 2).

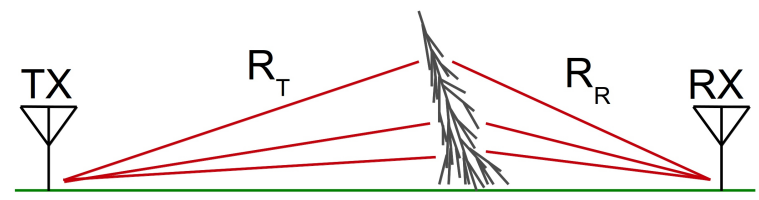

Figure 3: Bi-static geometry of a radar sounding wave interrogating an EAS to scale in the TARA geometry. $R_{T}$ and $R_{R}$ are the distances from transmitter (TX) to shower and shower to receiver (RX), respectively. The TX/RX antenna symbols represent location only. Actual antenna sizes are smaller than a pixel if represented to scale.

Chirp signals are ubiquitous in nature, although $\mathrm{CR}$ radar echos have very unique signatures. A simulation [23] has been designed that requires as inputs the CR energy, geometry and transmitter and receiver details, and which evolves an EAS according to standard particle production and energy transport models [22] while tracking the phase and amplitude of the radar echo. Shower parameters are functions of the primary particle energy [24]. The simulation indicates (see, for example, a "typical" TARA geometry simulation spectrogram in Figure 22 that CR radar echoes are short in duration (comparable to the shower life-time, $\approx 10 \mu \mathrm{s}$ ), have chirp rates of a few times $1 \mathrm{MHz} / \mu$ s and span a bandwidth on order of the sounding frequency (see Figures 4 and 5).

The energy and geometry of a distribution of 10000 cosmic rays detected at the TA surface detector array have been simulated. Figures 4 and 5 show distributions of the chirp rate and duration for these events. Data obtained from the simulation have been used to guide the design of the DAQ, transmitter system, and the receiver antennas. A 54.1 MHz radar sounding frequency (the TARA licensed frequency) implies the need to resolve a bandwidth of roughly $100 \mathrm{MHz}$ and therefore implement a DAQ with at least $200 \mathrm{MS} / \mathrm{s}$ ADC. An FPGA based design is necessary to implement real-time digital filters that will trigger the DAQ on signals that resemble chirp radar echos.

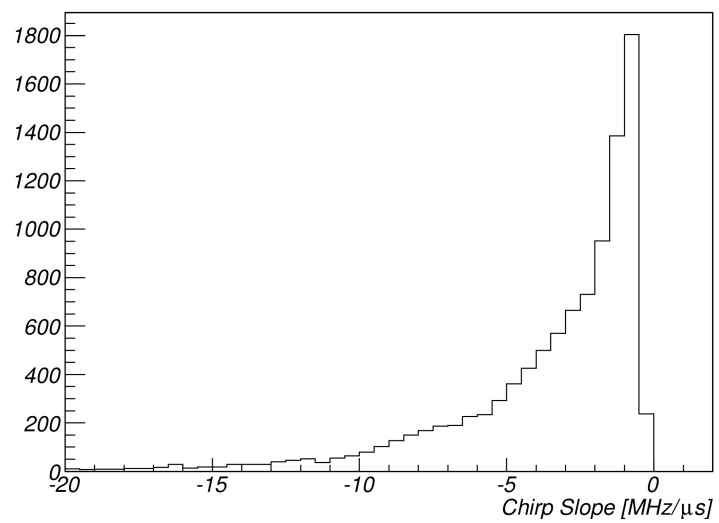

Figure 4: Simulated chirp rate distribution from a set of 10000 TA cosmic ray events. The rate is calculated from a weighted fit (by power) to the spectrogram of the simulated signal.

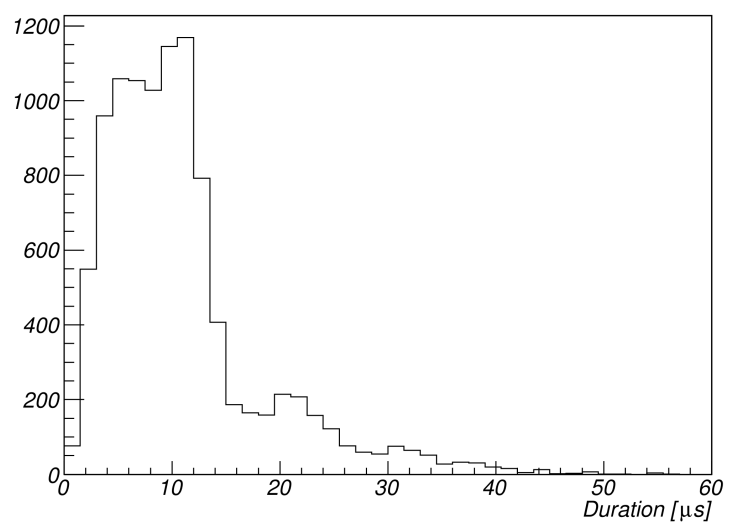

Figure 5: Chirp duration distribution from 10000 simulated radar echoes from TA cosmic ray events. Duration is defined as $d=t_{1}-t_{0}$, where $t_{0}$ is the time where the maximum power is received and $t_{1}$ is the later time when the received power drops by $20 \mathrm{~dB}$ below the maximum, which approximates the end of the shower.

Air showers are uniquely defined by their radar echo signatures with the exception of a lateral symmetry with respect to a plane connecting the transmitter and receiver and also a rotational symmetry about a line connecting the transmitter and receiver. Stereo detection is necessary (at minimum) to break this symmetry. Section 8 discusses the remote station prototype that will supplement our primary receiver for stereo detection. 
The actual radar cross section $\sigma_{E A S}$ is currently unknown. The bi-static radar equation gives the received power $P_{R}$ as a function of transmitter power $P_{T}$. Given the transmitter wavelength $\lambda$ and receiver and transmitter antenna gains $G_{R}$ and $G_{T}$, the bi-static radar equation is written as

$$
\frac{P_{R}}{P_{T}}=\left(\frac{G_{T}}{4 \pi R_{T}^{2}}\right) \sigma_{E A S}\left(\frac{G_{R}}{4 \pi R_{R}^{2}}\right) \frac{\lambda^{2}}{4 \pi} .
$$

Detection possibility depends on the signal-to-noise ratio (SNR), defined as

$$
\mathrm{SNR}=\frac{P_{\mathrm{c}}}{\sigma_{v}^{2}},
$$

where $P_{\mathrm{c}}$ is the chirp signal power and $\sigma_{v}$ is the standard deviation of the background noise. A second definition is necessary for signals with time-varying amplitude like those predicted by the EAS radar echo simulation. For such signals we use the amplitude signal-tonoise ratio (ASNR)

$$
\operatorname{ASNR}=\frac{v_{\max , \mathrm{c}}^{2}}{\sigma_{v}^{2}} .
$$

$v_{\max , \mathrm{c}}$ is the maximum chirp amplitude. The TARA DAQ can trigger on realistic chirp signals as low as $7 \mathrm{~dB}$ below the noise (-7 dB ASNR, see Section 7.4.2). A simple calculation will show that, if the thin wire approximation $\sigma_{t w}$ is assumed to correctly model the actual radar cross section (RCS) $\sigma_{E A S}$, TARA expects radar echoes with positive SNR (in $\mathrm{dB}$ ).

TARA detector parameters are given in Table 1 Consider a $60 \mathrm{MHz}$ Doppler shifted tone, scattered from an EAS located midway between the transmitter and receiver, which have a $39.5 \mathrm{~km}$ separation distance. Received power can be calculated from Equation 1 if $\sigma_{E A S} \simeq \sigma_{t w}$ is known. Some basic assumptions allow a quick calculation of $\sigma_{t w}$ : Shower $X_{\max }$ occurs roughly $2 \mathrm{~km}$ from the ground; the antennas' polarization vector and shower axis are in the same plane; the length $L$ of the scattering region of the shower is the speed of light multiplied by the electron attachment/recombination lifetime $\tau=10 \mathrm{~ns}$ [18], which implies $L=3 \mathrm{~m}$; the over-dense region radius near $X_{\max }$ is the thin wire radius [17] $a=0.01 \mathrm{~m}$. With these assumptions the thin-wire cross section [21] is $\sigma_{t w} \sim 1 \mathrm{~m}^{2}$ and the received power is $-79 \mathrm{dBm}$.

Section 5, Figure 20 shows a plot of receiver system background noise superimposed with galactic noise. Receiver sample rate and Fourier transform window size used in the calculation were $250 \mathrm{MS} / \mathrm{s}$ and 32768,

\begin{tabular}{|c|c|}
\hline Parameter & Value \\
\hline \hline UHECR energy & $10^{19} \mathrm{eV}$ \\
\hline$P_{T}$ & $40 \mathrm{~kW}$ \\
\hline$G_{T}$ & $22.6 \mathrm{dBi}($ Section 4.3$)$ \\
\hline$G_{R}$ & $12.6 \mathrm{dBi}($ Section 5$)$ \\
\hline$R_{T}=R_{R}$ & $19.75 \mathrm{~km}$ \\
\hline
\end{tabular}

Table 1: The list of the parameters assumed for calculating received power from a $60 \mathrm{MHz}$ Doppler shifted radar echo scattered from an EAS.

respectively. The power spectral density (PSD) of a $-79 \mathrm{dBm}$ tone detected by this system is $-79+$ $10 \log _{10}\left(32768 / 250 \cdot 10^{6}\right)=-117 \mathrm{dBm} / \mathrm{Hz}$. The reader should note that antenna patterns are both assumed to be at their maximum, which rarely occurs in practice. Further, polarization angle differences $(\phi)$ between the shower axis and antennas can yield another reduction in power $\propto \cos ^{4}(\phi)$.

The receiver background noise plot demonstrates that, in the TARA frequency band of interest, backgrounds are dominated by galactic noise. At $60 \mathrm{MHz}$, the background noise PSD is $-160 \mathrm{dBm} / \mathrm{Hz}$, much lower than that of a narrow-band Doppler shifted radar echo at $-117 \mathrm{dBm} / \mathrm{Hz}$ scattered from an ideal thin wire. Under reasonable assumptions for signal parameters, combined with our measured irreducible backgrounds and system response, the thin wire approximation for the radar cross-section $\sigma_{E A S}$ implies high values of signalto-noise.

\section{Transmitter}

\subsection{Hardware}

TARA operates a high power, Continuous Wave (CW), low frequency radar transmitter built from repurposed analog TV transmitter equipment with FCC call sign WF2XZZ, an experimental license. The transmitter site $\left(39^{\circ} 20^{\prime} 19.82400^{\prime \prime} \mathrm{N}, 112^{\circ} 42^{\prime} 3.24000^{\prime \prime} \mathrm{W}\right)$ is just outside Hinckley, UT city limits where human exposure to RF fields is of little concern. A high gain Yagi array (Section 4 focuses the radar wave toward the receiver station (Section 5) located $40 \mathrm{~km}$ away. Figure 1 shows the transmitter location near Hinckley and relative to the TA SD array [2]. The geometry was chosen to maximize the possibility of coincident SD and radar echo events.

Figure 6 shows a schematic of the transmitter hardware configuration. A Tektronix arbitrary function generator (AFG 3101; Tektronix, Inc.) provides the primary sine wave, which is amplified over nine orders of 


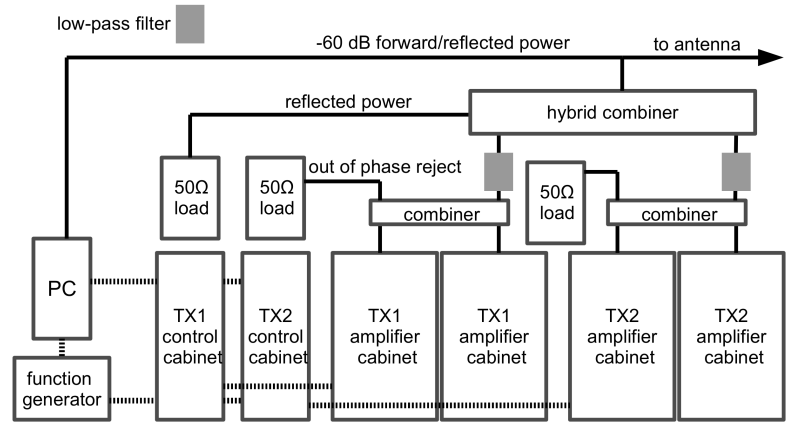

Figure 6: Schematic of the transmitter hardware configuration. A computer connected to RF sensor equipment, an arbitrary function generator and transmitter control electronics orchestrates the two distinct transmitters and provides remote control and logging. RF power from each transmitter's two amplifier cabinets is combined with out of phase power rejected into a $50 \Omega$ load. A hybrid combiner sums the combined output of each transmitter and sends that power to the antenna. Power reflected back into the hybrid combiner is directed to a third RF load.

magnitude before reaching the antenna. 54.1 MHz was chosen as the sounding frequency because of the lack of interference in the vacated analog channel two TV band and the $100 \mathrm{kHz}$ buffer between it and the amateur radio band which ends at 54.0 MHz.

Two $20 \mathrm{~kW}$ analog channel $2 \mathrm{TV}$ transmitters have a combined $40 \mathrm{~kW}$ power output. The primary signal from the function generator is split to feed both transmitters (Harris Platinum HT20LS, p/n 994-9236-001; Harris Broadcast) with the same level of gain. Each transmitter includes a control cabinet and two cabinets of power amplifier modules. RF power from each cabinet is combined in a passive RF combiner (620-2620002; Myat, Inc.) that routes any out-of-phase signal to a $50 \Omega$ load. The combined output of each transmitter is sent to a $90^{\circ}$ hybrid combiner (RCHC-332$6 \mathrm{LVF}$; Jampro, Inc.) that sums the total output of each transmitter. Between the final combined input and each transmitters' combined output there is an inline analog channel 2 low pass filter (visual low-pass filter, 3 1/8"; Myat, Inc.) to minimize harmonics. RF power leaves the building through $53 \mathrm{~m}$ of semi-flexible $31 / 8$ " circular air-dielectric wave guide (HJ8-50B; Andrew, Inc.).

Modifications were made to the transmitters to bypass interlocks that detect the presence of aural and visual inputs and video sync pulses necessary for standard TV transmission. Control cabinet electronics were calibrated to measure the correct forward and reflected power of the $54.1 \mathrm{MHz}$ tone instead of the RF envelope during the sync pulse. Currently, total power output is limited to $25 \mathrm{~kW}$ because of limitations that arise from amplifying a single tone versus the full $6 \mathrm{MHz}$ TV band.
Air conditioning and ventilation are critical to high power transmitter performance. Currently, transmitter efficiency is slightly better than $30 \%$, which implies that nearly $75 \mathrm{~kW}$ of heat must be removed from the building. The environment at the site is very dry and dusty, so all of the air brought into the building is filtered and positive gauge pressure is maintained. A single 25 ton $\mathrm{AC}$ unit filters and pumps cool air into the building. An economizer will shut down the compressor if the outside air temperature drops below $15.6^{\circ} \mathrm{C}\left(60^{\circ} \mathrm{F}\right)$. However, if the room is not cooling quickly with low outside ambient temperature, the compressor will be turned back on. Hot air near the ceiling is vented as necessary to maintain a slight positive pressure.

Future improvements to the transmitter will include biasing the power amplifiers for class B operation, in which amplification is applied to only half the $54.1 \mathrm{MHz}$ cycle. Resonance in the transmitter and antenna allow the second half of the wave to complete the cycle. Efficiency will nearly double compared with the current configuration.

\subsection{Remote Monitoring and Control}

Remote monitoring and control of the transmitter is important for two reasons. First, Federal Communications Commission (FCC) regulations require that nonstaffed transmitter facilities be remotely controlled and several key parameters monitored. Second, forward power and other parameters must be logged for receiver data analysis.

A computer interfaces with digital I/O and analog input devices that, in turn, are connected to the transmitters' built in digital I/O and analog output interface. RF power sensors (PWR-4GHS; Mini-Circuits) measure the final forward and reflected power via strongly attenuating sample ports on the wave guide near the building exit port. The sum of the two control cabinets' forward and reflected power measurements can be compared with the separate RF final forward and reflected power measurements.

The host computer monitors transmitter digital status, analog outputs and RF power sensors and controls the function generator. Logs are updated every five minutes with forward and reflected power for each transmitter, final (re: antenna) forward and reflected power, room temperature and various transmitter status and error states. Warning and error thresholds can trigger emails to the operators and initiate automatic shutdown. The program also provides a simple interface that allows the operator to remotely turn the transmitter on and off, increase or decrease forward power, and add a text log entry. 


\subsection{Performance}

TV transmitters are designed for $100 \%$ duty cycle operation. Similarly, the TARA transmitter is intended for continuous operation to maximize the probability of detection of UHECRs. With fixed gain and input signal, power is strongly correlated with transmitter room ambient temperature. Large temperature fluctuations in April 2013 resulted in a $\sim 3 \mathrm{~kW}$ spread in output power (Figure 7).

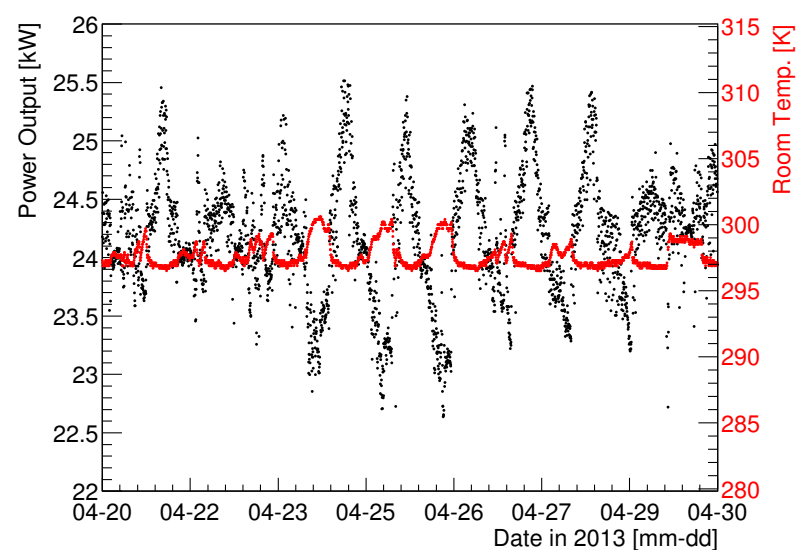

Figure 7: Transmitter forward power (black) and room temperature (red) during April 2013. Poor air conditioning calibration resulted in daily temperature fluctuations which caused large output power modulation.

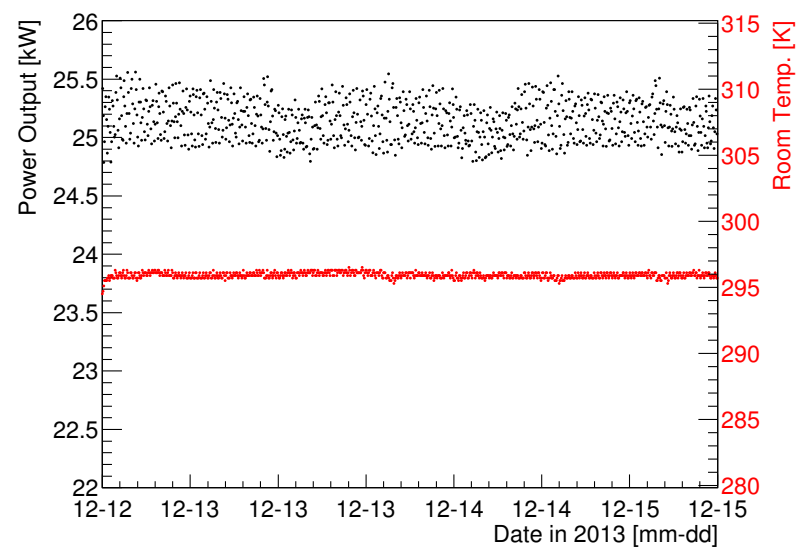

Figure 8: Transmitter forward power (black) and room temperature (red) during December 2013. A well-calibrated air conditioning system keeps room temperature stable and increased automatic gain control minimizes forward power fluctuations.

Transmitter forward power is more stable if room temperature is kept lower than $300 \mathrm{~K}\left(80^{\circ} \mathrm{F}\right)$. Figure 8 shows forward power fluctuations in August 2013 are much smaller than April. Built-in automatic gain control was increased during this period as well. The average power in December is higher than the average power in April because a slightly higher power input signal was used in later months. Reflected power is typically $\sim 100 \mathrm{~W}$, which is very low for such a high power system. This can be attributed to very good impedance matching with the extremely narrow-band Yagi antenna array.

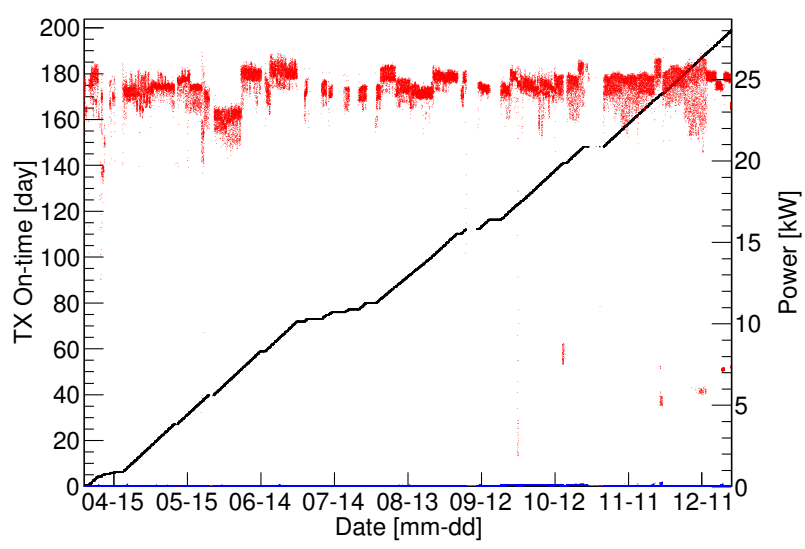

Figure 9: Transmitter on-time in days (black, left vertical axis) and forward and reflected power in units of $\mathrm{kW}$ (red and blue, right vertical axis) during 2013. Total duty cycle during this period is $83 \%$.

Figure 9 shows the total forward and reflected power in red and blue, respectively, referenced to the right vertical axis and the integrated on-time in black, referenced to the left vertical axis, since its commissioning in late March, 2013. The transmitter has been turned off several times for maintenance and testing and during periods when our receiver equipment was removed from the field for upgrades. Although forward power is not continuous and fluctuations were large in the past, we consider 200 days of operation in the first year to bode well for future data collection.

Harmonics have been measured to confirm compliance with FCC regulations and to avoid interfering with other stations. With total forward output at $25 \mathrm{~kW}$, the fundamental and several harmonic frequencies were measured from a low power RF sample port. The first five harmonics are about $60 \mathrm{~dB}$ below the fundamental (see Table 2). Harmonics will be further attenuated by about $30 \mathrm{~dB}$ by the intrinsic bandpass of the antenna. 


\begin{tabular}{|l|c|}
\hline Frequency $(\mathrm{MHz})$ & Power $(\mathrm{dBm})$ \\
\hline \hline 54.1 & 8.5 \\
\hline 108.2 & -66.0 \\
\hline 162.3 & -68.3 \\
\hline 216.4 & -84.4 \\
\hline 270.5 & $-89^{*}$ \\
\hline 324.6 & $-77^{*}$ \\
\hline 378.7 & $-94^{*}$ \\
\hline 432.8 & $-87^{*}$ \\
\hline 486.9 & $-98^{*}$ \\
\hline 541.0 & $-91^{*}$ \\
\hline
\end{tabular}

Table 2: Power of fundamental frequency and first ten harmonics for the 54.1 MHz radar sounding wave. These measurements were taken from a highly attenuated final forward power RF sample port. Total transmitted power was approximately $25 \mathrm{~kW}$. FM and TV stations are required by the FCC to limit the first ten harmonics to at least $60 \mathrm{~dB}$ below their approved total transmitted power. Experimental station $\mathrm{WF} 2 \mathrm{XZZ}$ is exempt from this requirement although it readily meets it. (*fluctuating value, $\pm 5 \mathrm{~dB}$ )

\section{Transmitting Antenna}

\subsection{Physical Design}

As the bi-static radar equation (Equation 1) shows, the received power is the product of the scattering cross section, transmitted power, transmitter antenna gain, receiver antenna gain and receiver aperture. Because the physics of the radar scattering cross section is not well understood, an antenna with high gain and directivity was chosen to maximize received power.

The TARA transmitting antenna is composed of 8 narrow band Yagi antennas designed and manufactured by M2 Antenna Systems, Inc. Each Yagi is constructed of aluminum and capable of handling $10 \mathrm{~kW}$ of continuous RF power. The specifications for each Yagi are a frequency range of $53.9-54.3 \mathrm{MHz}, 12 \mathrm{dBi}$ free space gain, front to back ratio of $18 \mathrm{~dB}$, and beamwidths (defined as the angle in the plane under consideration over which the radiated power is within three $\mathrm{dB}$ of the maximum) of $27^{\circ}$ and $23^{\circ}$ in the vertical and horizontal planes respectively.

Each Yagi antenna is composed of five elements: a reflector, driven element, and three directors, and are mounted on a $21.6 \mathrm{ft}$ long, 2 " diameter boom. A balanced t-match is fed from a 4:1 coaxial balun which transforms the unbalanced $50 \Omega$ input to the balanced $200 \Omega$ used to drive the antenna. A $50 \Omega 7 / 8^{\prime \prime}$ coaxial waveguide connects the balun to the four port power dividers. Table 3 describes the lengths and positions of the antenna elements on the boom. All elements are constructed of aluminum tubing of $3 / 4^{\prime \prime}$ outer diameter. Each element, except for the driven element is constructed of two equal sections that are joined at the boom via $7 / 8^{\prime \prime}$ outer diameter sleeve elements. The weight is $35 \mathrm{lbs}$ when completely assembled.

\begin{tabular}{|l|r|r|}
\hline Element & Length (in) & Position (in) \\
\hline \hline Reflector & 107.625 & -44.375 \\
\hline Driven Element & 100.500 & 0.000 \\
\hline Director 1 & 99.500 & 51.125 \\
\hline Director 2 & 97.250 & 131.625 \\
\hline Director 3 & 97.000 & 193.625 \\
\hline
\end{tabular}

Table 3: Length and relative boom position of antenna elements of the TARA Yagi antennas. All elements have a diameter of $0.75^{\prime \prime}$.

Transmitter output power is delivered to the antenna array via approximately 100 feet of CommScope HJ850B $31 / 8^{\prime \prime}$ Heliax air dielectric coaxial wave guide. The Heliax then connects to a two port power divider located at the base of the antenna array. Each output port of the power divider feeds equal length $15 / 8^{\prime \prime}$ coaxial cables, which in turn feed a four port power divider. Each four port power divider then delivers power to the individual Yagi antennas via equal length $7 / 8^{\prime \prime}$ coaxial cables. All components in the transmission line chain are impedance matched to $50 \Omega$.

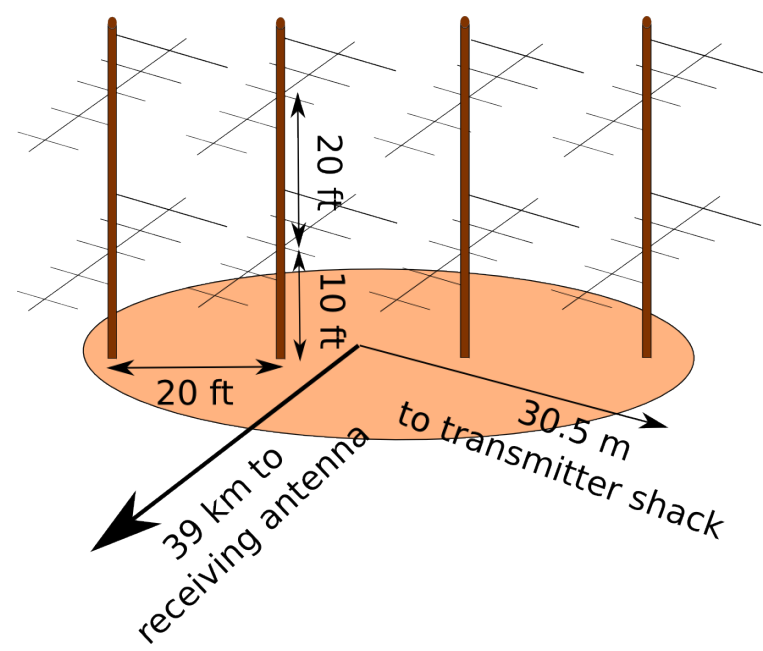

Figure 10: Configuration of the eight Yagi antennas and mounting poles which comprise the TARA transmitting antenna array.

The antennas are mounted on four wooden telephone poles, two stacked vertically on each pole. The bottom and top antennas on each pole are located $10 \mathrm{ft}$ and $30 \mathrm{ft}$ above the ground respectively. Currently, the antennas are mounted in a configuration that provides a horizontally polarized signal. Wooden poles were used to allow 
a change of polarization. The poles, separated by $20 \mathrm{ft}$, are aligned in a plane perpendicular to the line pointing toward the receiver site located at the Long Ridge fluorescence detector $39 \mathrm{~km}$ to the southwest. Figure 10 shows the antenna array configuration.

\subsection{Theoretical Performance}

The eight Yagi antennas are operated as a phased array to take advantage of pattern multiplication to improve gain and directivity relative to the individual antennas. The design philosophy of the antenna array is to deliver a large amount of power in the forward direction in a very narrow beam to maximize the power density over the TA surface detector. High power density is equivalent to a large $P_{T} G_{T}$ factor in the bi-static radar equation, which is needed to increase the chance of detection of a cosmic ray air shower via radar echo given the uncertainty in the radar scattering cross section $\sigma_{E A S}$. Before construction, modeling of the array was performed using version two of NEC [25], an antenna modeling and optimization software package.

Figure 11 shows the radiation pattern of the full eight Yagi array when configured as shown in Figure 10 Forward gain is $22.6 \mathrm{dBi}$, horizontal beam width is $12^{\circ}$, vertical beam width is $10^{\circ}$, the front-to-back $(\mathrm{F} / \mathrm{B})$ ratio is $11.8 \mathrm{~dB}$ and the elevation angle of the main lobe is $9^{\circ}$.

Simulations were performed to find the best spacing between the mounting poles, vertical separation of antennas and height above ground to shape and direct the main lobe in a preferred direction. Antenna pole spacing influences the main lobe beam width. A narrower beam width can be obtained at the expense of transferring power to the side lobes which do not direct RF energy over the TA surface detector. Elevation angle is manipulated by antenna height above ground. Changing this parameter does little else to the main lobe. Elevation angle and beam width were selected to increase the probability that air shower $X_{\max }$ would fall in the path of the main lobe where the charged particle density is the greatest. The $9^{\circ}$ main lobe elevation angle is chosen such that the sounding wave illuminates the mean $X_{\max }$ midway between transmitter and receiver for a distribution of showers (varying zenith angle) of order $10^{19} \mathrm{EeV}[26]$.

\subsection{Measured Performance}

The ability of an antenna to transmit energy is best characterized by the reflection coefficient $S_{11}$ (also called return loss when expressed in $\mathrm{dB}$ ). It is a measure of the ratio of the voltage reflected from a transmission line relative to input. Large reflection coefficient implies
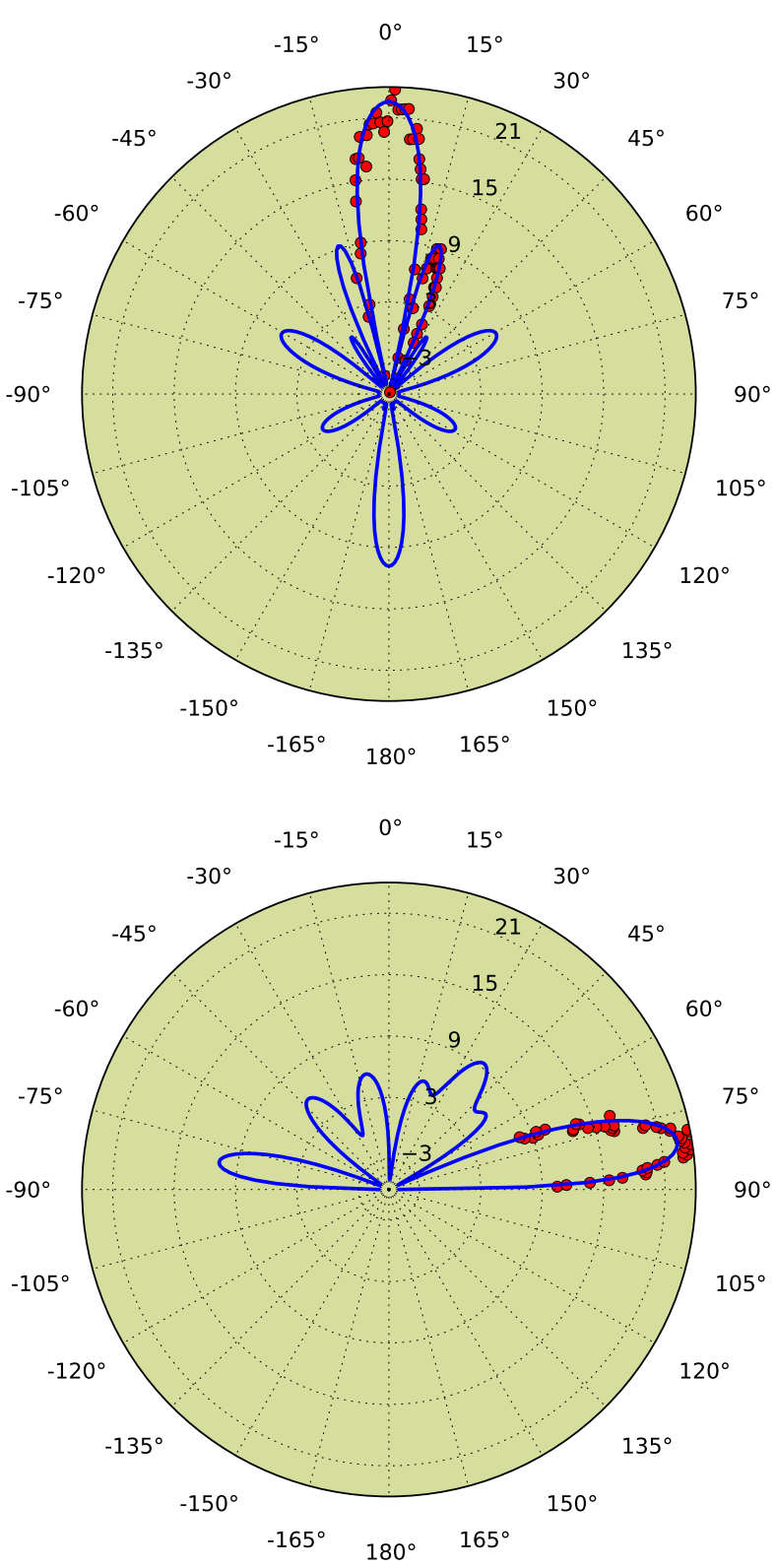

Figure 11: Simulated horizontal (top) and vertical (bottom) radiation patterns of the eight Yagi TARA antenna array shown in blue. Red points are measured data that have been uniformly scaled to best fit the model. Forward gain is $22.6 \mathrm{dBi}$, beam width is $12^{\circ}$ horizontal, $10^{\circ}$ vertical, and the $\mathrm{F} / \mathrm{B}$ ratio is $11.8 \mathrm{~dB}$.

significant energy is reflected back into the transmitter building which can interfere with other electronics, elevate ambient temperature and even damage the transmitter. Figure 12 shows the reflection coefficient for the Yagi array. It shows a return loss of $-37.25 \mathrm{~dB}$ at the sounding frequency, which is excellent. $S_{11}$ of $-20 \mathrm{~dB}$ 


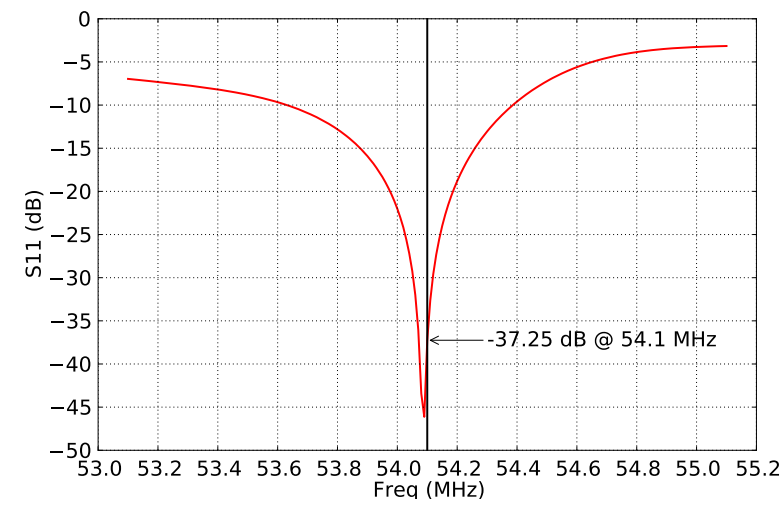

Figure 12: Reflection coefficient $\left(S_{11}\right)$ for the eight Yagi array.

or less is considered good.

To verify that the transmitting antenna is operating as designed, an RF power meter or similar device can be used to measure the power as a function of position relative to the antenna. This measurement is challenging because it must be performed in the far field of the antenna (typically $r \gg \lambda$ ). To fully probe the radiation pattern of the TARA transmitting antenna, power measurements must be made high above the ground since the main lobe is inclined $9^{\circ}$ relative to horizontal.

Vertical radiation pattern measurements were taken by using antenna transmitting/receiving symmetry. A tethered weather balloon was floated with a custom battery powered $54.1 \mathrm{MHz}$ signal generator that fed a dipole antenna. Over a range of discrete heights, received power was recorded at the output (normally the input) of the Yagi array.

The horizontal (azimuthal) radiation pattern was measured using a spectrum analyzer on the ground to determine the pointing direction and shape of the main lobe. Measurements of transmitted RF power were taken at distances between 650 and $1000 \mathrm{~m}$ radially from the center of the array. Power was measured along a road that does not run perpendicular to the pointing direction of the transmitter so a $1 / r^{2}$ correction was made. Figure 11 shows the measured points for the horizontal and vertical patterns overlayed on the models. These measurements are all relative, not absolute, so a uniform scale factor was determined by minimizing $\chi^{2}$ between the model and data. The measured pattern agrees very well with the model in pointing direction and shape.

\section{Receiver Antenna}

The TARA receiver antenna site is located at the Telescope Array Long Ridge Fluorescence Detector

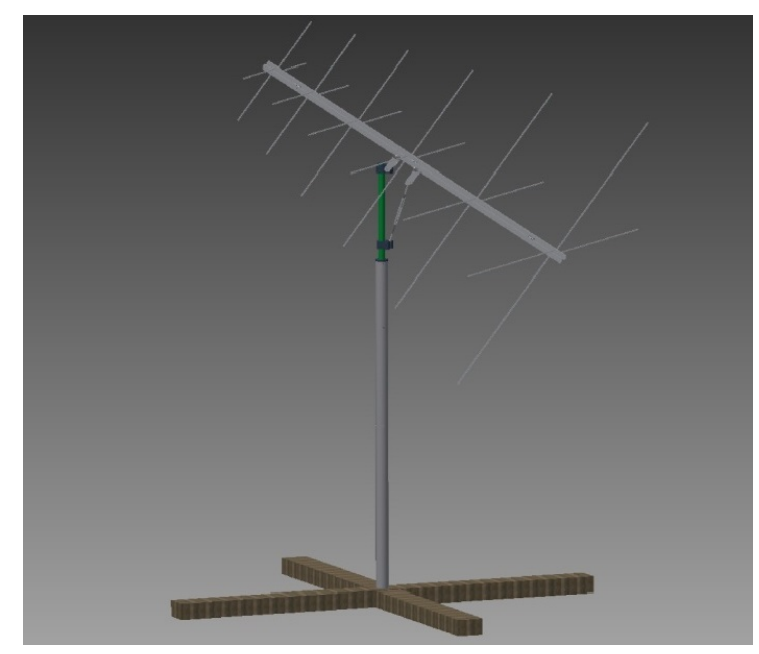

Figure 13: Dual polarized TARA Log Periodic Dipole Antenna (LPDA).

\begin{tabular}{|l|r|r|}
\hline Element & Length (in) & Position (in) \\
\hline \hline 1 & 21.875 & 3.625 \\
\hline 2 & 26.625 & 18.0625 \\
\hline 3 & 32.5 & 35.625 \\
\hline 4 & 39.625 & 57.0 \\
\hline 5 & 48.3125 & 83.125 \\
\hline 6 & 58.3125 & 115.0 \\
\hline
\end{tabular}

Table 4: Length and relative boom position of antenna elements of the TARA Log Periodic Dipole Antennas. All elements have a diameter of $0.25^{\prime \prime}$.

$\left(39^{\circ} 12^{\prime} 27.75420^{\prime \prime} \mathrm{N}, 113^{\circ} 7^{\prime} 15.56760^{\prime \prime} \mathrm{W}\right)$. Receiver antennas are dual-polarized log periodic dipole antennas (LPDA) designed to match the expected $<100 \mathrm{MHz}$ signal frequency characteristics. Due to noise below $30 \mathrm{MHz}$ and the FM band above $88 \mathrm{MHz}$, the effective band is reduced to 40 to $80 \mathrm{MHz}$. Each antenna channel is comprised of a series of six $\lambda / 2$ dipoles. The ratio of successive dipole lengths is equal to the horizontal spacing between two dipoles (the defining characteristic of LPDA units), with the longest elements farthest from the feed-point to mitigate large group delay across the passband. Table 4 gives the lengths and positions of the antenna elements on the boom from the front edge to the back. All elements are constructed of aluminum tubing of $1 / 4^{\prime \prime}$ outer diameter. Figure 13 shows a schematic of the receiver LPDA.

The impedance of the antenna against a $50 \Omega$ transmission line was measured in an anechoic chamber at the University of Kansas. The standing wave ratio (SWR), the magnitude of the complex reflection coefficient $\left(S_{11}\right)$, is shown as a function of frequency in Fig- 


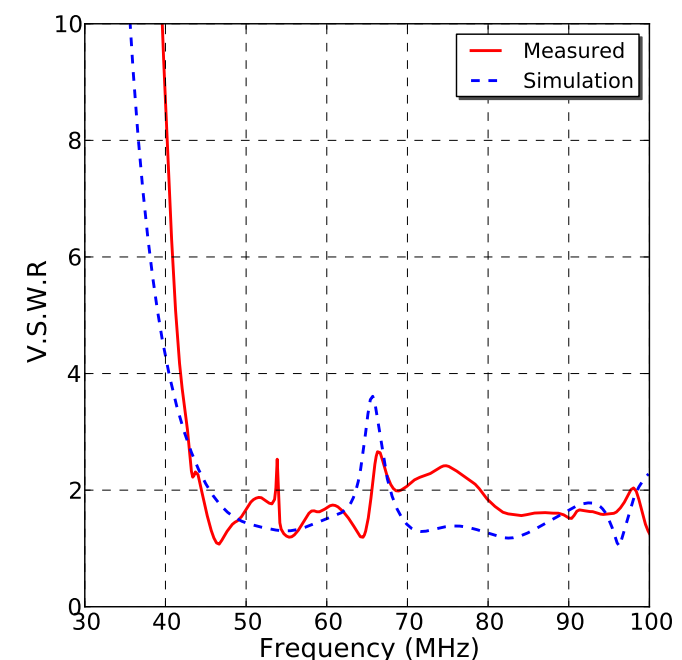

Figure 14: SWR of a horizontally polarized TARA LPDA as measured in an anechoic chamber.

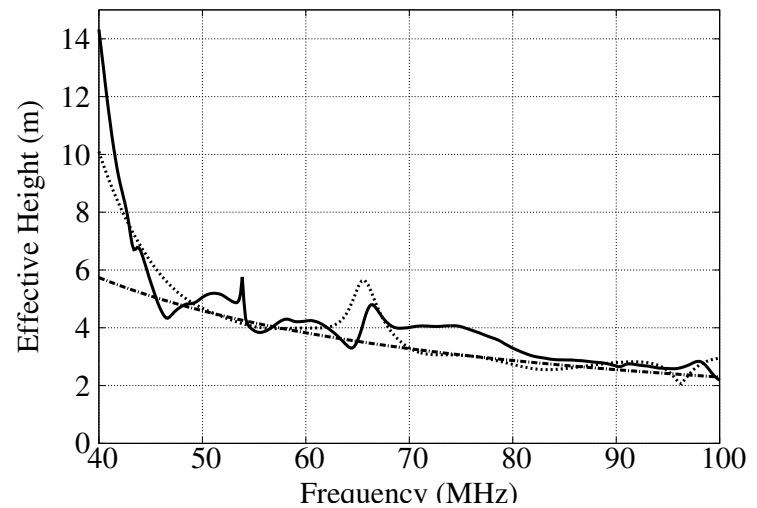

Figure 15: Effective height in meters vs. frequency in $\mathrm{MHz}$ of the TARA receiver LPDA. The $S_{11}$ parameter and gain of the receiver antenna are inserted into Equation 4 and plotted vs. frequency using the anechoic chamber data (solid line), simulated data from NEC (fine dashed), and simulated data with the $54.1 \mathrm{MHz}$ values of $S_{11}$ and gain held constant (dot-dashed line).

ure 14 An SWR of 3.0 implies greater than $75 \%$ signal power is transmitted from the antenna to the receiver at a given frequency.

The complex $S_{11}$ measurement also quantifies the effective height of the LPDA. The effective height translates the incident electric field strength in $\mathrm{V} / \mathrm{m}$ to a voltage at the antenna terminals. It is given as $\mathbf{E}_{\text {inc }} \cdot \mathbf{h}_{\mathbf{e f f}}=$ $\left|E_{\text {inc }} \| h_{\text {eff }}\right| \cos (\theta)=V$, where $\theta$ is the polarization angle and the antenna is assumed to be horizontally polarized. The boresight effective height can be expressed [27] as
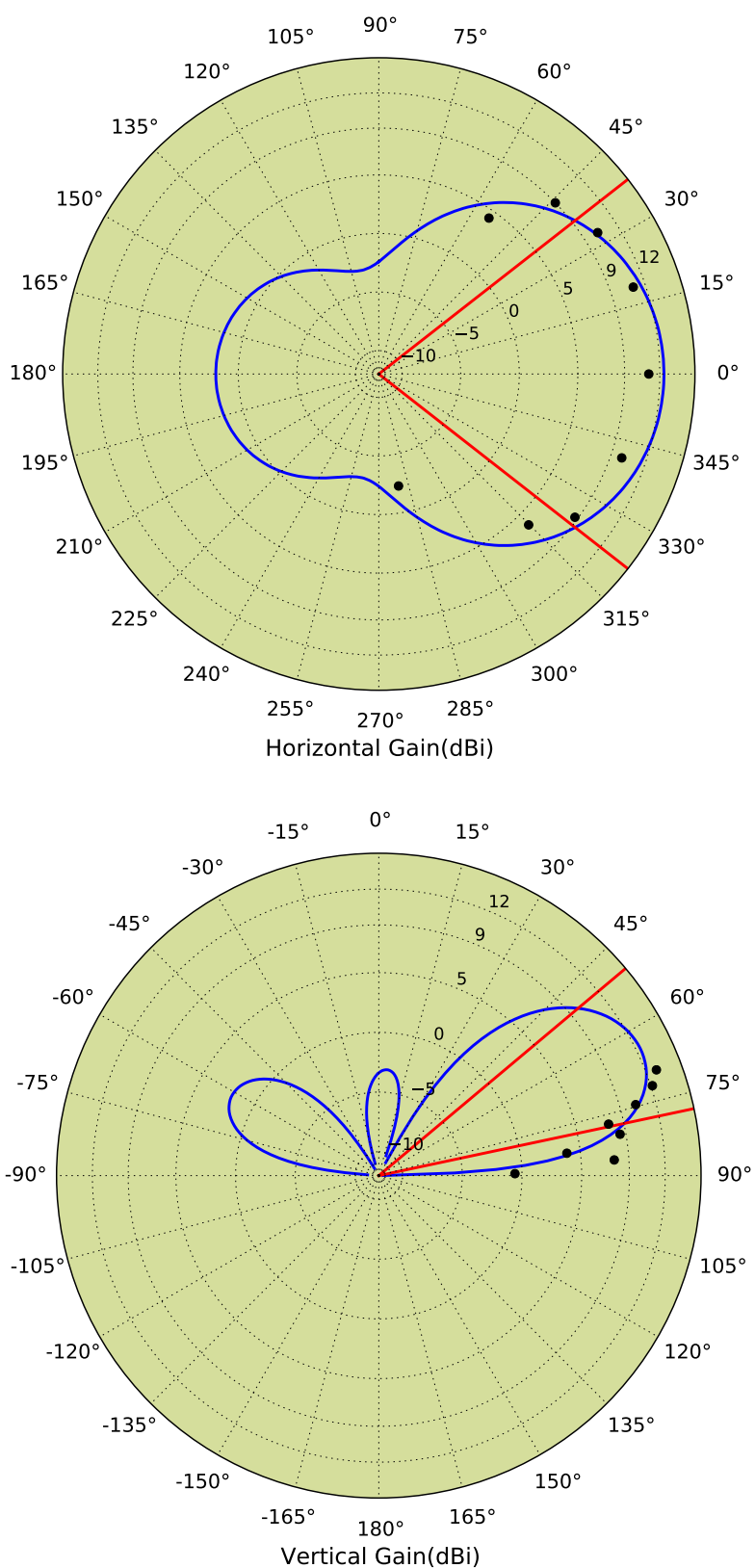

Figure 16: Simulated horizontal (top) and vertical (bottom) radiation pattern of a horizontally polarized TARA LPDA at the transmitter sounding frequency of 54.1 MHz. Beamwidths $(-3 \mathrm{~dB}$ below peak gain) are shown with red lines. Peak gain is $12.6 \mathrm{dBi}$.

$$
h(v)=2 * \sqrt{\frac{G c^{2}\left|Z_{i n}\right|}{4 \pi v^{2} Z_{0}}} .
$$

In the effective height expression, $G$ is the measured gain of $12.6 \mathrm{dBi}$ (see Figure 16, $c$ is the speed of light, 
$Z_{\text {in }}$ is the complex antenna impedance, $v$ is the frequency, and $Z_{0}=120 \pi$ is the impedance of free space. In terms of the measured complex reflection coefficient $S_{11}$, the impedance is given by $\left|Z_{i n}\right|=\left|\frac{1+S_{11}}{1-S_{11}}\right| 50 \Omega$. The frequency-dependent magnitude of the effective height is plotted in Figure 15 .

Receiver antenna gain is a factor in the bi-static radar equation that affects detection threshold. NEC was used in simulating the radiation pattern of the antenna to confirm directionality (see Figure 16). Simulated forward gain is $12.6 \mathrm{dBi}$ and the vertical beamwidth is $23^{\circ}$ at the carrier frequency, 54.1 MHz. Figure 17 displays measured beamwidth in the band of interest.

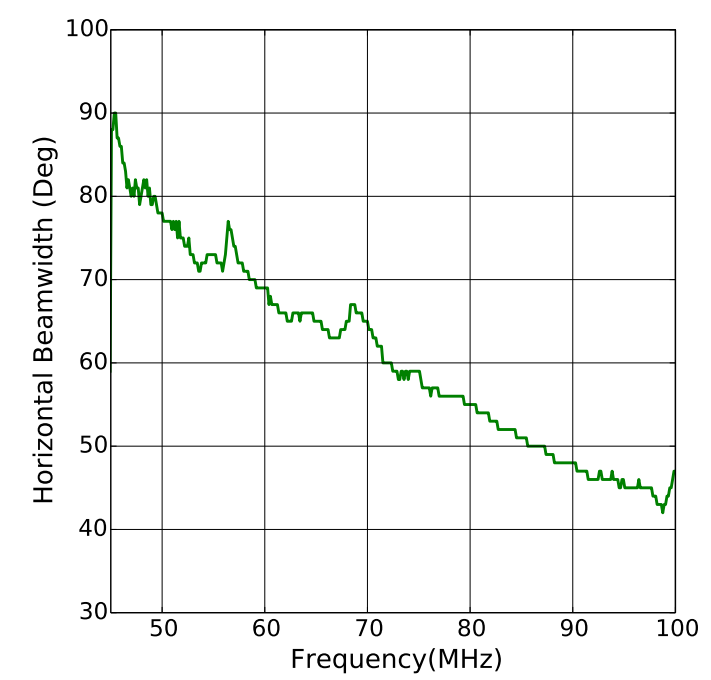

Figure 17: Beamwidth of a single channel LPDA as measured in an anechoic chamber at the University of Kansas.

\section{Receiver Front-end}

There are three dual-polarization antennas at the receiver site, two of which are currently connected to the DAQ (Section 7). RF signal from the antennas pass through a bank of filters and amplifiers. The components include an RF limiter (VLM-33-S+; MiniCircuits), broad band amplifier, low pass filter (NLP 100+; Mini-Circuits), high pass filter and an FM band stop filter (NSBP-108+; Mini-Circuits). Both polarizations from one antenna are filtered $(37 \mathrm{MHz}$ cutoff frequency high pass filter, SHP-50+; Mini-Circuits) and amplified (40 dB, ZKL-1R5+; Mini-Circuits) at the antenna, where a bias tee (ZFBT-4R2G+; Mini-Circuits) is used to bring DC power from the control room.
The second antenna's channels are filtered $(25 \mathrm{MHz}$ high pass filter, NHP-25+; Mini-Circuits) and amplified (30 dB, ZKL-2R5+; Mini-Circuits) inside the control room. The lightning arrester (LSS0001; Inscape Data) minimizes damage to sensitive amplifiers by electric potentials that accrue during thunderstorms. The RF limiter prevents damage by transient high amplitude pulses (see Section 7.2).

Signal conditioning in the amplifier/filter banks is characterized by the transmission coefficient (Figure 18) $S_{21}$. It is a measure of the ratio of the voltage at the end of a transmission line relative to the input. Impedance mismatch relative to a $50 \Omega$ transmission line, insertion loss for the various devices and gain from the amplifiers are combined in $S_{21}$ data. Of note in Figure 18 is the flat, high-gain $(30 \mathrm{~dB})$, broadband $(\simeq 40 \mathrm{MHz})$ passband necessary for Doppler-shifted radar echoes.

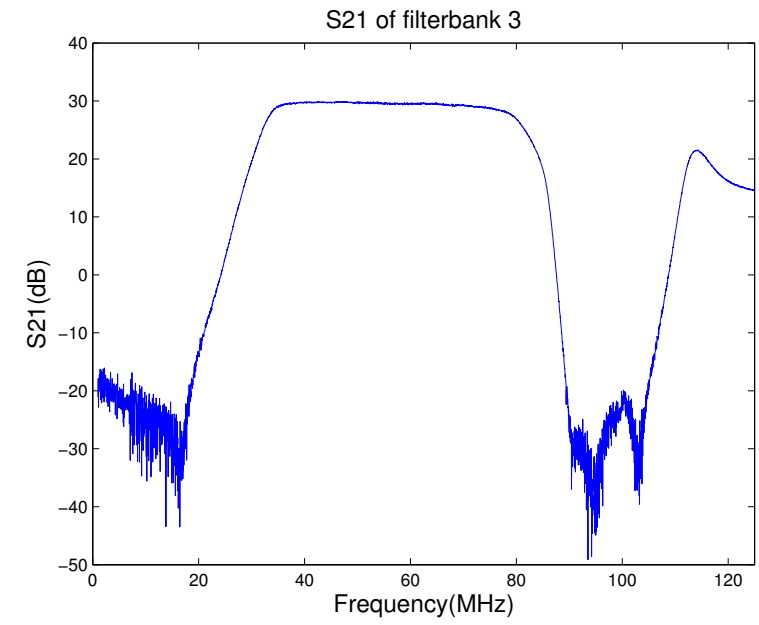

Figure 18: $S_{21}$ (transmission coefficient) of the filter and amplifier bank connected to the triggering channel of the DAQ.

In any RF receiver system, sensitivity is limited by the combination of external noise entering through the antenna and internal noise from various sources like low noise amplifiers and other resistive losses from filters, cables and couplers. Noise entering the antenna is generated by the sky, earth and antenna resistive loss. Diffuse radio noise from the galactic plane is non-polarized and is the dominant noise source in the TARA frequency band. Figure 19 shows diurnal variation in the snapshot (forced trigger, $1 \mathrm{~min}^{-1}$ ) spectrum that remains consistent in data taken six months apart. Each plot shows the Power Spectral Density (PSD, units of $\mathrm{dBm} / \mathrm{Hz}$ ) averaged over eight days versus Local Mean Sidereal Time (LMST). Horizontal and vertical error bars are bin width and std. dev. of the mean, respectively. The effect 

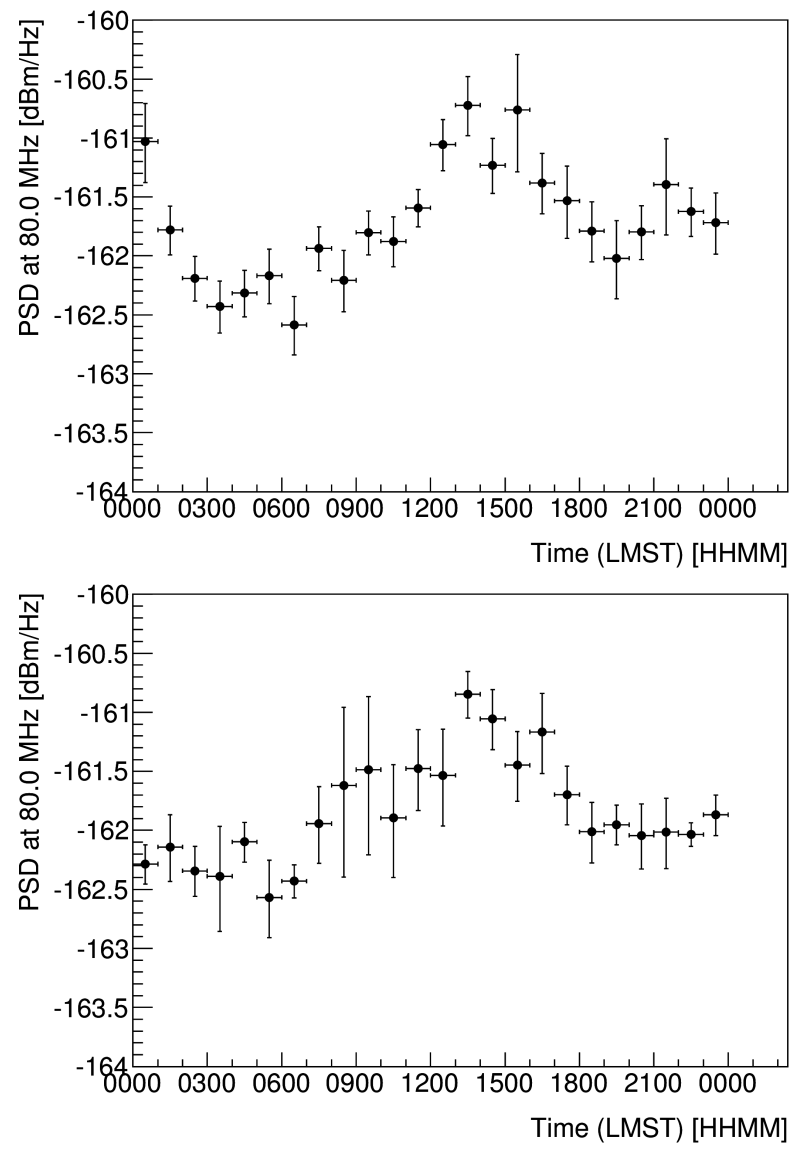

Figure 19: Snapshot (forced trigger) Power Spectral Density (PSD) at 80.0 MHz averaged over eight days versus Local Mean Sidereal Time (LMST). Top: Data taken in December, 2013. Bottom: Data taken in May, 2014. There is strong correlation in peak PSD and sidereal time which indicates the signal is galactic in origin. Horizontal error bars show bin width. Vertical error bars are std. dev. in the mean.

of amplifiers and cable losses have been removed such that absolute received power is shown. Data taken in December, 2013 are shown in the top plot, with those recorded in May, 2014 shown in the bottom plot. We observe that the peak occurs at the same time and power in each plot. Our conclusion is diurnal fluctuations are caused by changing perspective on the galactic center.

By accounting for amplifier and instrumental gains and losses, the observed noise background can be compared with the irreducible galactic noise background [29] across the passband. Our average measured system noise is calibrated by removing the effects of individual components in the receiver RF chain from average snapshot spectra to determine the absolute received power. Without any other scaling, our corrected received power compares nicely with the galactic noise standard [28] (Figure 20]. Important components for

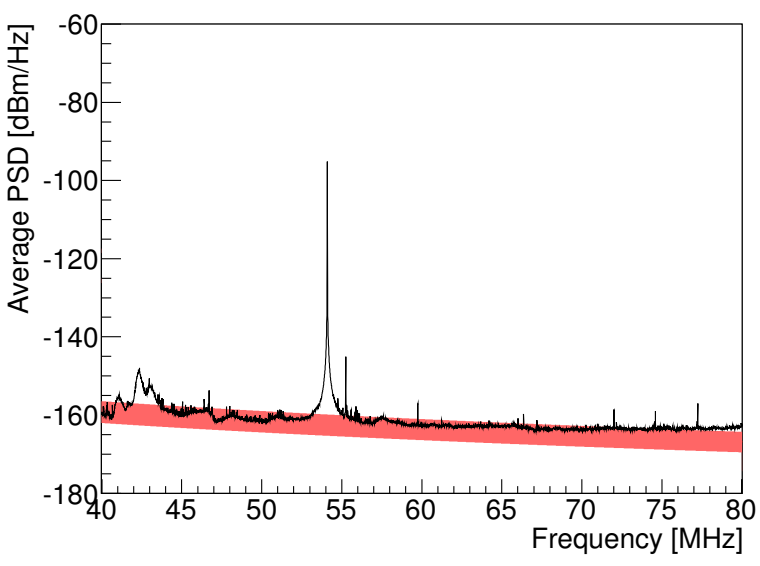

Figure 20: Average receiver system noise floor (black) Power Spectral Density (PSD) in $\mathrm{dBm} / \mathrm{Hz}$ superimposed with a fit to measured galactic background noise and its associated error [28] (red band). System attenuation, filters and amplifiers were accounted for to determine absolute received power. No other calibration or scaling was applied to the receiver data.

which adjustments were made include filters and amplifiers via the measured transmission coefficient $S_{21}$ and LMR-400 transmission line with attenuation data. Anthropogenic noise sources are transient and stationary noise is absent within our measurement band due to the receiver site's remote location. In this frequency region, galactic noise dominates thermal and other noise sources.

\section{Receiver DAQ}

\subsection{DAQ Structure}

The National Instruments FlexRIO system provides an integrated hardware and software solution for a custom software defined radio DAQ. It is composed of three basic parts: adapter module, FPGA module and host controller (as shown in the lower box of Figure 21). A description of each of these subsystems follows.

The NI-5761 RF adapter module is a highperformance digitizer that defines the physical inputs and outputs of the DAQ system. It digitizes four analog input channels at a rate of $250 \mathrm{MS} / \mathrm{s}$ with 14-bit resolution. Eight TTL I/O lines are available for additional control, some of which are used in custom DAQ triggering schemes.

The NI-7965R FPGA module is based on the PXI express platform which uses a Xilinx Virtex-5 FPGA with $128 \mathrm{MB}$ on board DRAM. FPGA design provides accurate timing and intelligent triggering. The PXI-express platform has a high-speed data link to the host controller, which is connected to the development machine, 


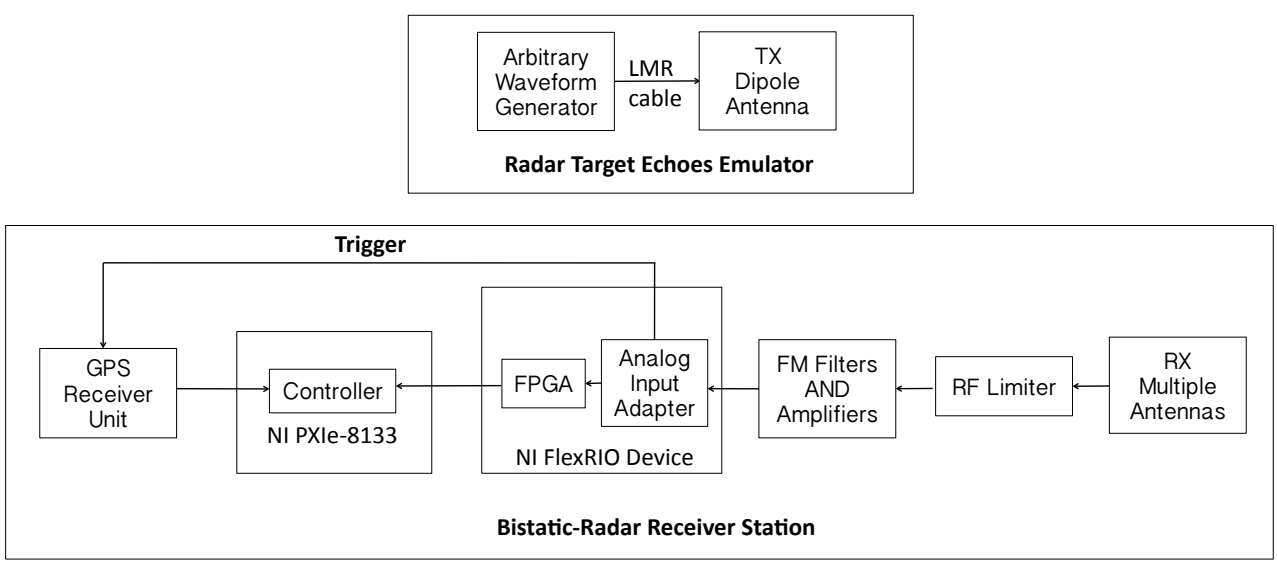

Figure 21: Elements of the radar receiver station.

a Windows based computer, which uses the LabVIEW environment to design and compile FPGA code. A host controller application, also designed in LabVIEW, runs on the development machine.

\subsection{Design Challenges}

Based on the high velocity of the radar target, echoes are excepted to be characterized by a rapid phase modulation-induced frequency shift, covering tens of $\mathrm{MHz}$ in $10 \mu \mathrm{s}$. As the magnitude of the Doppler blue shift decreases as the shower develops in the atmosphere, these signals sweep (approximately) linearly from high to low frequency and are categorized as linear-downward chirp signals. Echo parameters are dependent on the physical parameters of the air showers. Thus, unlike existing chirp applications, we are interested in the detection of chirp echoes of variable amplitude, center frequency and frequency rates within a relatively wide band. In addition, the detection threshold must be minimized in order to increase the probability of detecting radar echoes with SNR less than one.

Furthermore, UHECR events are rare and random in time. TA receives only several $>10^{19} \mathrm{eV}$ events per week, so background noise and spurious RF activity dominate.

Figure 22 shows a spectrogram of data acquired in the field using the complete receiver and test system (Figure 21, where FM radio and noise below $\sim 30 \mathrm{MHz}$ are filtered out. The time-frequency representation shows that the background noise of our radar environment is rich with multiple undesirable components including stationary tones outside the $40-80 \mathrm{MHz}$ effective band located at $28.5 \mathrm{MHz}$ and, inside the band, the carrier at $54.1 \mathrm{MHz}$ as well as broadband transients. Sudden amplitude modulation of stationary sources and powerful, short-duration broadband noise can cause false-alarms. A robust signal processing technique is needed to confront these challenges [30].

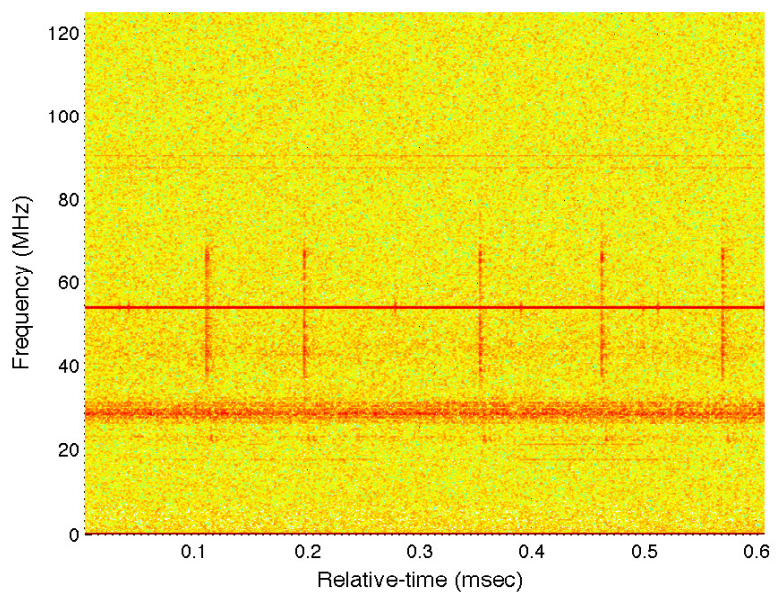

Figure 22: Spectrogram of background noise at the receiver site. Frequency and time are on the vertical and horizontal axes, respectively, with color representing the power in a particular frequency component. The carrier signal is represented by the horizontal line at 54.1 MHz. Broadband transients are the vertical lines and stationary noise sources are the horizontal band near $30 \mathrm{MHz}$.

\subsection{DAQ Implementation}

The DAQ is designed to detect chirp echoes and confront the problem of a variable noise environment. Two antennas feed the DAQ's four input channels. Each antenna is a dual-polarized LPDA (Section 5) with one output channel each for horizontal and vertical polarization. Data are collected simultaneously from each of the four analog channels with one horizontal channel 
considered the triggering channel, then sampled using a $250 \mathrm{MS} / \mathrm{s}$ ADC (Texas Instruments; ADS62P49). Ana$\log$ to digital conversion is followed by fast digital memory storage on the FPGA chip, which stores the incoming samples from each channel sequentially, in a $131 \mu \mathrm{s}$ (32744 sample) continuous circular buffer such that data in each buffer are continually overwritten. Three distinct trigger modes are implemented: "snapshot", "Fluorescence Detector (FD) external", and "matched-filter bank".

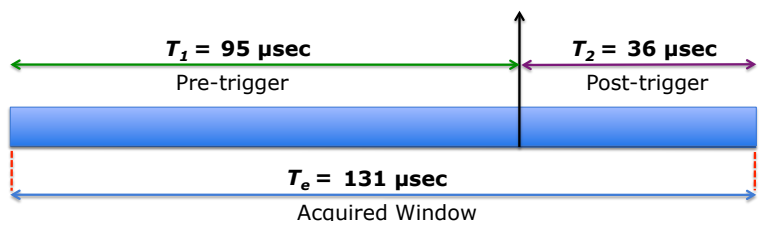

Figure 23: Position of the triggering pulse within the data window that is written to disk.

When a trigger occurs, the circular buffer information is sent to the host controller to be permanently stored on the computer's disk. A $320 \mu$ s dead-time is required to transfer data from a buffer to FPGA memory, during which the DAQ cannot accept triggers. Sustained maximum trigger rate is $50 \mathrm{~Hz}$ due to FPGA-to-host data transfer limitations. As depicted in Figure 23 pre/post trigger acquisition is set to $95 \mu \mathrm{s}$ and $36 \mu \mathrm{s}$, respectively, to allow for delay and jitter in the FD trigger timing (33 $\mu$ s delay, $1 \mu \mathrm{s}$ jitter) and sufficient post-trigger data to see an entire echo wave form. A GPS time stamp is retrieved from a programmable hardware module [31] and recorded for each trigger with an absolute error of $\pm 20 \mathrm{~ns}$

The snapshot trigger is an unbiased trigger scheme initiated once every minute that writes out an event to disk. These events will (likely) contain background noise only. Unbiased triggers are crucial for background noise estimation and analysis.

During active FD data acquisition periods, the Long Ridge FD (the location of the TARA receiver site) emits a NIM (Nuclear Instrumentation Module) pulse for each low level trigger with a typical rate of $\sim 3-5 \mathrm{~Hz}$ or much higher during FD calibration periods. The low level trigger is an OR of individual FD telescope mirror triggers. Dead time due to high FD-trigger rates are as high as several milliseconds during calibration periods. This does not reduce data acquisition time significantly because these periods occur only for several minutes and less than half a dozen times per FD data acquisition period. Further, FD operation only amounts to $10 \%$ duty cycle on average. The FlexRIO is forced to trigger by each pulse received from the FD. Each FD run will result in many thousands of triggers which can be narrowed to several events that coincide with real events found in reconstructed TA data.

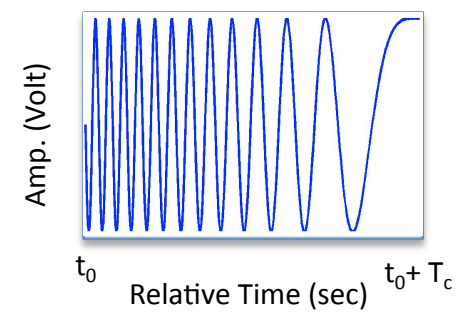

(a)

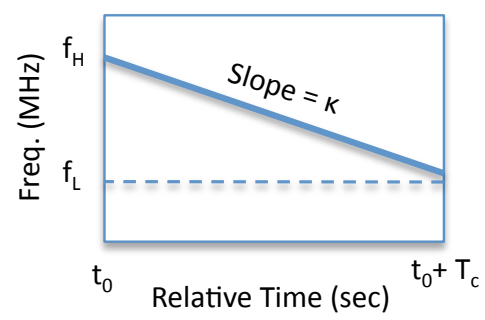

(b)

Figure 24: Linear down-chirp signal. (a) Signal in time-domain. (b) Signal in time-frequency domain.

The matched filter (MF) bank is a solution for the problem of detecting radar chirp echoes in a challenging noise background using signal processing techniques. The signal of interest is assumed to be a down-chirp signal that has duration $T_{c}$ seconds with a constant amplitude, start (high) frequency $f_{\mathrm{H}}$, center frequency $f_{\mathrm{C}}$, end (low) frequency $f_{\mathrm{L}}$ and chirp rate $\kappa \mathrm{Hz} / \mathrm{sec}$. An example of the signal of interest is shown in Figure 24 Assuming that it is centered around time $t=0$, such a chirp signal is written as

$$
s(t)=\operatorname{rect}\left(\frac{t}{T_{c}}\right) \cos \left(2 \pi f_{\mathrm{C}} t-\pi \kappa t^{2}\right) .
$$

where $\operatorname{rect}(x)$ is the rectangle function and $t$ is the time in seconds.

We limit our interest to detecting the presence of $s(t)$ within a certain bandwidth, without prior knowledge of the chirp rate $\kappa$. Based on simulation of the physical target, reflected echoes are expected to have a peak amplitude within or near the range [60-65] MHz. Thus, we consider $f_{\mathrm{H}}$ to be $65 \mathrm{MHz}$ and $f_{\mathrm{L}}$ to be $60 \mathrm{MHz}$.

Since the chirp rate varies, we use a bank of filters matched to a number of quantized chirp rates, $\kappa_{1}, \kappa_{2}$, 
$\cdots, \kappa_{M}$. A functional block diagram of the detection process is illustrated in Figure 25.

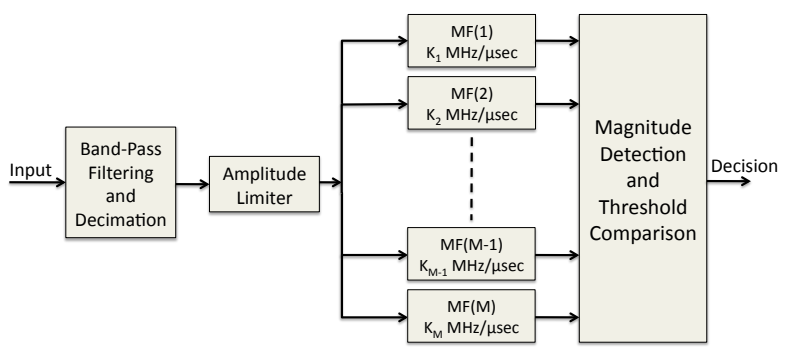

Figure 25: Block diagram of the matched-filter-type detector.

Let $\mathbf{y}_{m}$ denote the output samples of the $m$ th matched filter and $\gamma_{m}$ the threshold at the filter output. As depicted in Figure 25, a trigger decision is made at the output of the matched-filter bank by comparing magnitudes of the elements of $\mathbf{y}_{\mathbf{1}}, \mathbf{y}_{\mathbf{2}}, \cdots, \mathbf{y}_{\mathbf{M}}$, each, against the corresponding threshold levels $\gamma_{1}, \gamma_{2}, \cdots, \gamma_{M}$, respectively.

Threshold levels are defined as $n_{\gamma}$ units of the signal level (equivalently, noise standard deviation) at the output of each filter, denoted by $\sigma_{m}$ for the $m$ th matched filter. Every time a trigger condition (the presence of a chirp) is met, an event is declared. Since the background noise level varies with time, $\sigma_{m}$ is measured every five seconds to maintain a constant data acquisition rate.

The most probable chirp-rate interval for a distribution of simulated radar echoes is $\mathcal{K}=[-3,-1] \mathrm{MHz} / \mu \mathrm{s}$. We choose $M=5$ and the chirp rates (in $\mathrm{MHz} / \mu \mathrm{s}$ ) as

$$
\begin{gathered}
\kappa_{1}=-1.1161, \kappa_{2}=-1.3904, \kappa_{3}=-1.7321, \\
\kappa_{4}=-2.1577, \kappa_{5}=-2.6879 .
\end{gathered}
$$

\subsubsection{Amplitude Limiter}

Radio background at the remote receiver site is clear of stationary interference signals in the frequency band of interest, 40-80 MHz. Therefore, the broadband transients mentioned in Section 7.2 are the primary source of false alarms. Consequently, the threshold of the MF detector must be raised in order to maintain the desired false alarm rate. The result is high data rate in return for low trigger thresholds. A digital amplitude limiter applied immediately before the input to the MF detector helps to minimize false alarms while keeping the detection threshold as low as possible and without significantly degrading detection efficiency.

The amplitude limiter clips the amplitude of the received signal to a fraction $k$ of its RMS value before clipping. Its mathematical expression is

$$
\left\{\begin{array}{l}
y=x, \quad|x|<k \sigma_{s} \\
y=k \sigma_{s}, \quad x>k \sigma_{s} \\
y=-k \sigma_{s}, \quad x<-k \sigma_{s} .
\end{array}\right.
$$

where $x$ is the raw input, $y$ is the amplitude limited output, and $\sigma_{s}$ is the RMS value of the signal before clipping. The result is a reduced relative power ratio of the spurious impulses to the non-perturbed background. Clipping also lowers the waveform RMS in proportion to the clipping level.

\subsubsection{Band-Pass Filtering}

We observe considerable CW noise within the 40$80 \mathrm{MHz}$ band, including the carrier signal. The carrier and other persistent tones can have large amplitudes and lead to high matched filter RMS output which can, as shown in the next section, prevent detection of low SNR chirp signals. Such tones, including the carrier, can be easily filtered out. Before the amplitude limiter, a narrow band-pass filter eliminates all frequencies outside a $60-65 \mathrm{MHz}$ band with $-80 \mathrm{~dB}$ stop band attenuation. Data stored in the ring buffer are not filtered this way.

\subsection{Performance Evaluation}

Detection performance of the MF detector has been evaluated under two test signal conditions: noise only or signal plus noise. For each test, the Boolean result of the threshold comparison with the MF outputs is recorded. The probability of signal plus noise exceeding MF thresholds is the efficiency and the average rate of erroneous detection decisions caused by filtered noise is false alarm rate.

The ability to detect a received chirp signal in background noise depends on the ratio of the signal power to the background noise power. Radar carrier power dominates the background so two quantities are used to describe the background noise. First, we define the ratio of the test chirp signal power to the radar carrier power as the signal-to-carrier ratio (SCR). Second, we use either the SNR (Equation 2) or ASNR (Equation 3), depending on the type of test chirp signal input to the matched-filter bank, after filtering out the powerful carrier signal.

Consider the following observations about performance analysis. First, it is clear that system performance depends on the chosen threshold level $n_{\gamma}$ (user defined, a multiple of $\sigma_{m}$ as defined previously) for each SNR value. False alarm rate is expected to decrease as the threshold level increases, at the expense 
of detection efficiency of low SNR chirp signals. Conversely, detection efficiency increases as the threshold decreases. Second, the false alarm rate is expected to decrease as the amplitude limiter level decreases because high amplitude transients are effectively removed. To this date, radar echoes from CR air showers have not been detected, so it's unlikely that the EAS cross section is large enough to produce such large amplitude impulses. Therefore such signals are dismissed a priori. Our strategy is to choose the threshold and amplitude limiter level that gives high detection efficiency for a given SNR and low false alarm rate.

Two tests are conducted to determine the ideal amplitude limiter level and the efficiency as a function of MF threshold. The goal of the first test is to measure the average false alarm rate of the non-Gaussian noise environment and evaluate the improvement that could be achieved by adding the amplitude limiter. Results are shown in Figure 26 for three different amplitude limiter levels, which clearly show that the limiter level has a significant effect on the false alarm rate. Efficiency curves for different amplitude limiter levels (described in the next paragraphs) show that the amplitude limiter does not decrease detection performance of chirp signals, although they are also clipped.

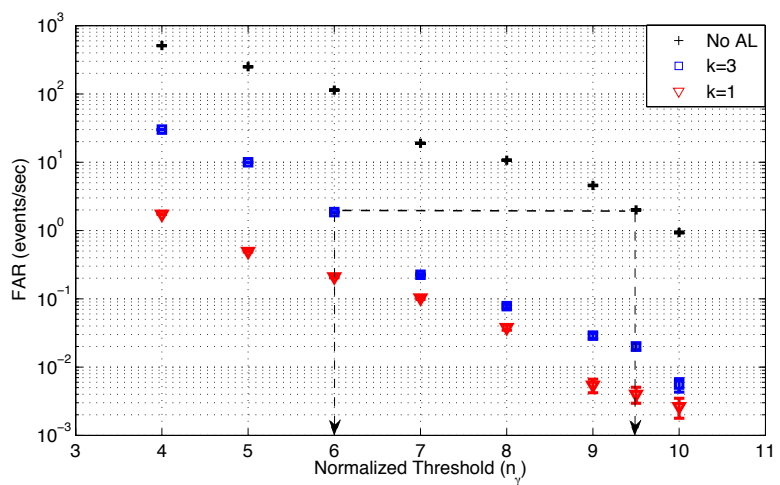

Figure 26: False-Alarm Rate versus relative threshold ( $n_{\gamma}$ units of the standard deviation at each filter output) for different amplitude limiter levels.

Consider the following interpretation of Figure 26. In order to achieve a $2 \mathrm{~Hz}$ false alarm rate, $n_{\gamma}$ has a value of six for $k=3$ and 9.5 for $k=10$ (black dashed line). Thus, detection thresholds can be decreased which enhances positive detection of low SNR signals.

The second test applies a theoretical chirp signal with various chirp rates and SNR values that correspond to a reasonable false alarm rate. Based on data storage and post-processing computational requirements, we have decided that a false alarm rate of $\sim 1 \mathrm{~Hz}$ is reasonable.
Artificially generated chirp signals are transmitted in situ to the receiving antennas by an arbitrary waveform generator (AFG 3101; Tektronix, Inc.) and a dipole antenna. Both linear chirp signals and a simulated radar echo (see Section 2) are used in measuring detection performance.

\subsubsection{Linear chirp signal}

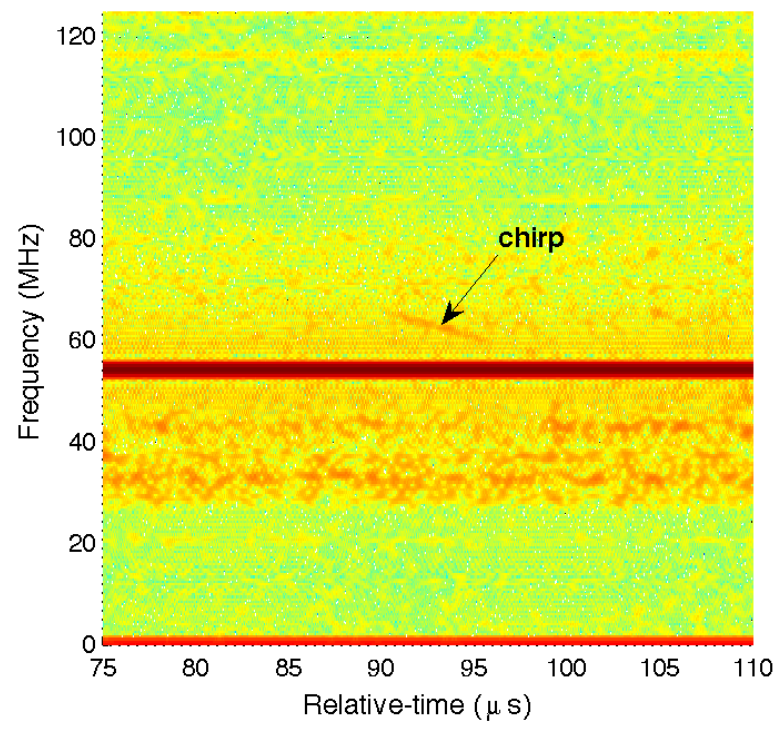

Figure 27: Time-frequency (spectrogram) representation of a linear, $-1 \mathrm{MHz} / \mu \mathrm{s},-10 \mathrm{~dB} \mathrm{SNR}$ received chirp signal as recorded by the DAQ system.

A periodic, linear chirp with $-1 \mathrm{MHz} / \mu$ s rate is embedded in a real receiver site background wave form. Figure 27 shows the spectrogram of a chirp embedded with $-10 \mathrm{~dB}$ SNR and $-40 \mathrm{~dB}$ SCR value.

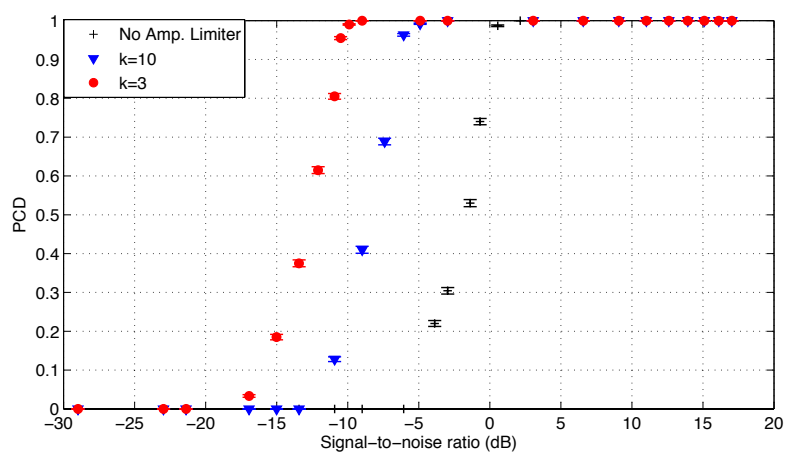

Figure 28: Probability of detection for the matched-filter-type detector with $n_{\gamma}=6$.

Figure 28 shows detection performance for a $2 \mathrm{~Hz}$ false alarm rate. Efficiency is shown for cases where the 
amplitude limiter is removed and at two different levels that result in the same false alarm rate, each with different threshold levels. The minimum SNR for which complete detection is achieved is $5 \mathrm{~dB}$ when no amplitude limiter is applied, $0 \mathrm{~dB}$ for $k=10$ (soft clipping), $-6 \mathrm{~dB}$ for $k=3$ (hard clipping). These results imply that by using the amplitude limiter, high detection performance can be achieved with low complexity. To maximize detection ability, the amplitude limiter is currently fixed at $k=3$.

\subsubsection{Simulated Air Shower}

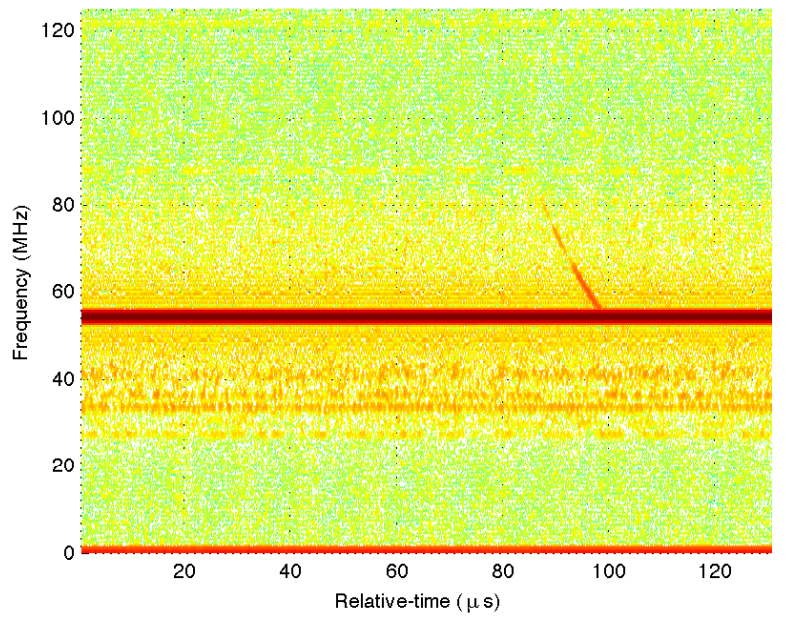

Figure 29: Spectrogram of simulated air shower radar echo with $5 \mathrm{~dB}$ ASNR. The radar echo is from a simulated shower inclined $30^{\circ}$ out of the $T X \rightarrow R X$ plane and located midway between the transmitter and receiver.

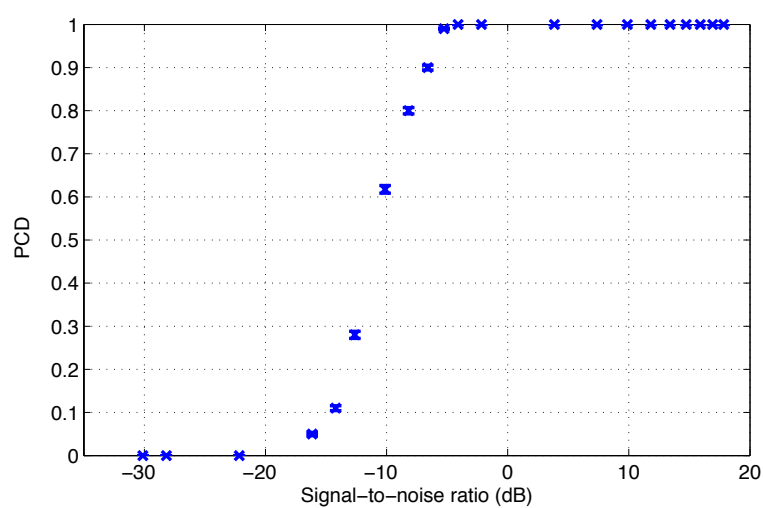

Figure 30: Probability of correct detection for the matched-filter detector using $n_{\gamma}=6$ for a simulated air-shower echo that is scaled and emulated with a function generator.

In a more realistic test, a simulated radar echo from a $10 \mathrm{EeV}$ air shower inclined $30^{\circ}$ out of the $T X \rightarrow R X$ plane and located midway between the transmitter and receiver is scaled and transmitted to the receiving antennas using a function generator. Figure 29 shows a spectrogram of the received waveform with $5 \mathrm{~dB}$ ASNR and $-25 \mathrm{~dB}$ SCR. The echo is broadband (about $25 \mathrm{MHz}$ ) and short in duration $(10 \mu \mathrm{s})$. Detection efficiency of the emulated chirp is shown in Figure 30 The minimum ASNR for which complete detection is achieved is $-7 \mathrm{~dB}$.

\section{Remote Receiver Station}
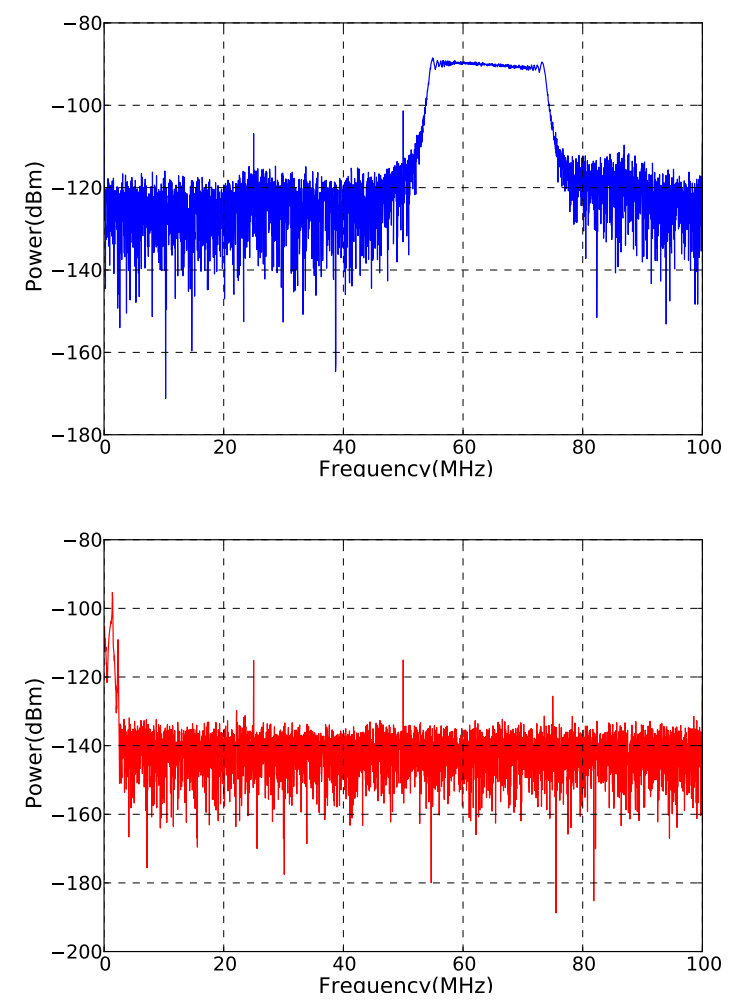

Figure 31: Top: the power spectrum of a $-10 \mathrm{MHz} / \mu$ s chirp created by a signal generator, prior to mixing. Bottom: the power spectrum of a $1 \mathrm{MHz}$ monotone signal after signal mixing and passing through a low pass filter. The chirp is evident as the left-most peak in this distribution.

In addition to signal detection using matched filtering in the FlexRIO, an alternative technique has been developed that accomplishes chirp detection using a primarily analog signal chain. Remote stations, by definition, are generally subject to less radio interference, and add stereoscopic measurement capabilities which theoretically allow unique determination of CR geometry and core location. In contrast to the FlexRIO system, a mostly analog data acquisition system has lower power 
consumption at a cost which is also comparatively inexpensive. Triggering logic for our remote receiver station and some specific details of hardware components are discussed in the next couple subsections.

\subsection{Remote Triggering}
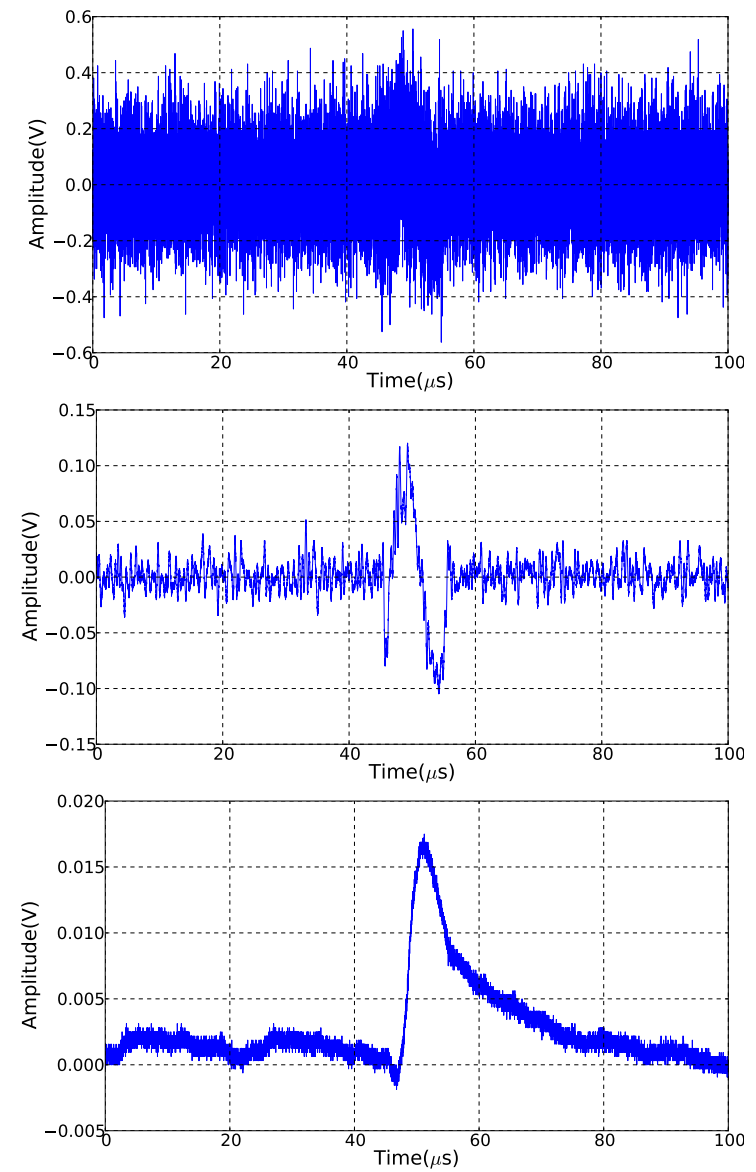

Figure 32: Top: A $0 \mathrm{~dB}$ SNR and $1 \mathrm{MHz} / \mu$ s chirp embedded in noise prior to mixing. Second from top: The monotone signal after input chirp is mixed with delayed copy of itself and passed through a lowpass filter. Bottom: Monotone passed through the Agilent 8471D power detector.

The alternative approach is based on an analog frequency mixer. The input signal is mixed with a delayed copy of itself, i.e, $s(t) \otimes s(t-\tau)$. For an incident chirp signal, the non-linear components in the mixer result in a product term that yields a monotone at a beat frequency $f_{\text {beat }}=\kappa \tau$; dependent only on the delay time $\tau$ and the chirp rate $\kappa$. The delay is created with $100 \mathrm{ft}$ of LMR-600 cable, which produces negligible losses and removes the need for power consuming active components. With appropriate filtering, the problem of chirp detection is ultimately reduced to that of detecting a down-converted monotone. This is illustrated in Figure 31. Portrayed here with an oscilloscope is a $-10 \mathrm{MHz} / \mu$ s chirp which has been converted to a $1 \mathrm{MHz}$ monotone by mixing with a delayed copy of itself.

After mixing, the signal is passed through an envelope detector (8471D; Agilent, Inc.). The entire time domain signal chain is illustrated in Figure 32. In this oscilloscope based example, a chirp with $0 \mathrm{~dB}$ SNR at a rate of $-1 \mathrm{MHz} / \mu \mathrm{s}$ is first band-pass filtered (41$100 \mathrm{MHz}$ ) and then amplified by $20 \mathrm{~dB}$. The signal is then mixed and low-pass filtered (DC-1.9 MHz) and passed through the Agilent power detector.

The expected value of chirp rates from EAS echos is typically between -1 to $-10 \mathrm{MHz} / \mu$ s (see Section 2). Consequently, with 100 ns delay, the down-converted signal has a frequency between $100 \mathrm{kHz}$ and $1 \mathrm{MHz}$. To trigger on such signals, the mixed signal is split into multiple copies. Each copy is then passed through custom band-pass filters and an envelope detector. Different frequency bands are then compared by majority logic in an FPGA, requiring no more than one band to form a trigger in order to suppress impulsive noise. Each of the frequency banded outputs corresponds to a separate range of chirp rates. The block diagram in Figure 33 outlines this triggering procedure.

\subsection{Remote Station Electronics}

The layout of the full remote station, currently in the field outside of Delta, UT, including the Chirp Acquisition Module (CAM), power systems, acquisition electronics, and communications blocks is shown in Figure 34

The Chirp Acquisition Module has a modular design enabling quick debugging of the constituent components. This unit is comprised of a custom triggering board encompassing four band pass filters and envelope detectors, and a four channel ADC (AD80066; Analog Devices) with 16 bit resolution sampling at $4 \mathrm{MS} / \mathrm{s}$ per channel to sample the signal out of the envelope detectors. A high speed ADC (AD9634 evaluation board; Analog Devices) sampling at $200 \mathrm{MS} / \mathrm{s}$ directly samples the raw data from the Antenna and an FPGA (Spartan - 6 LX16; Xilinx) performs the majority comparison logic to trigger and capture triggered data before transferring to a single board computer. A Raspberry Pi (Rev. 2) single board computer stores triggered data in an SD card along with GPS time stamps (M12M; iLotus).

Another major component is the System Health Monitor [32] (SHM), which both monitors performance and 


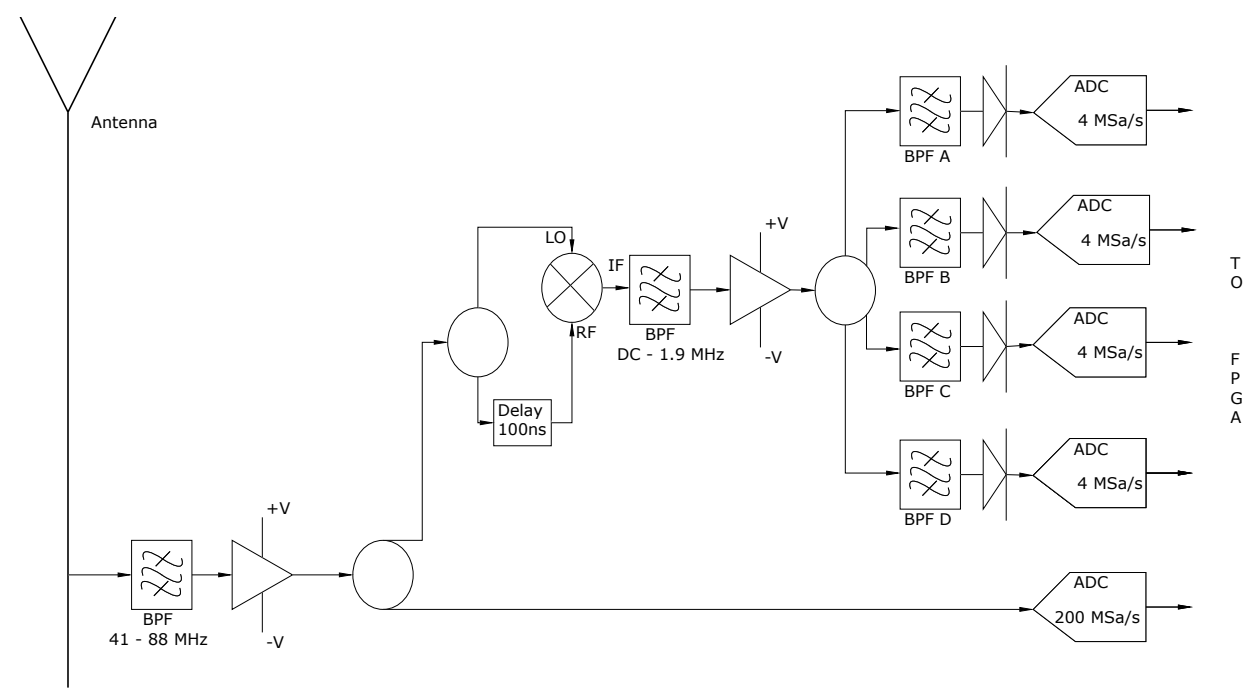

Figure 33: Block diagram of the event triggering to be employed in the remote station.

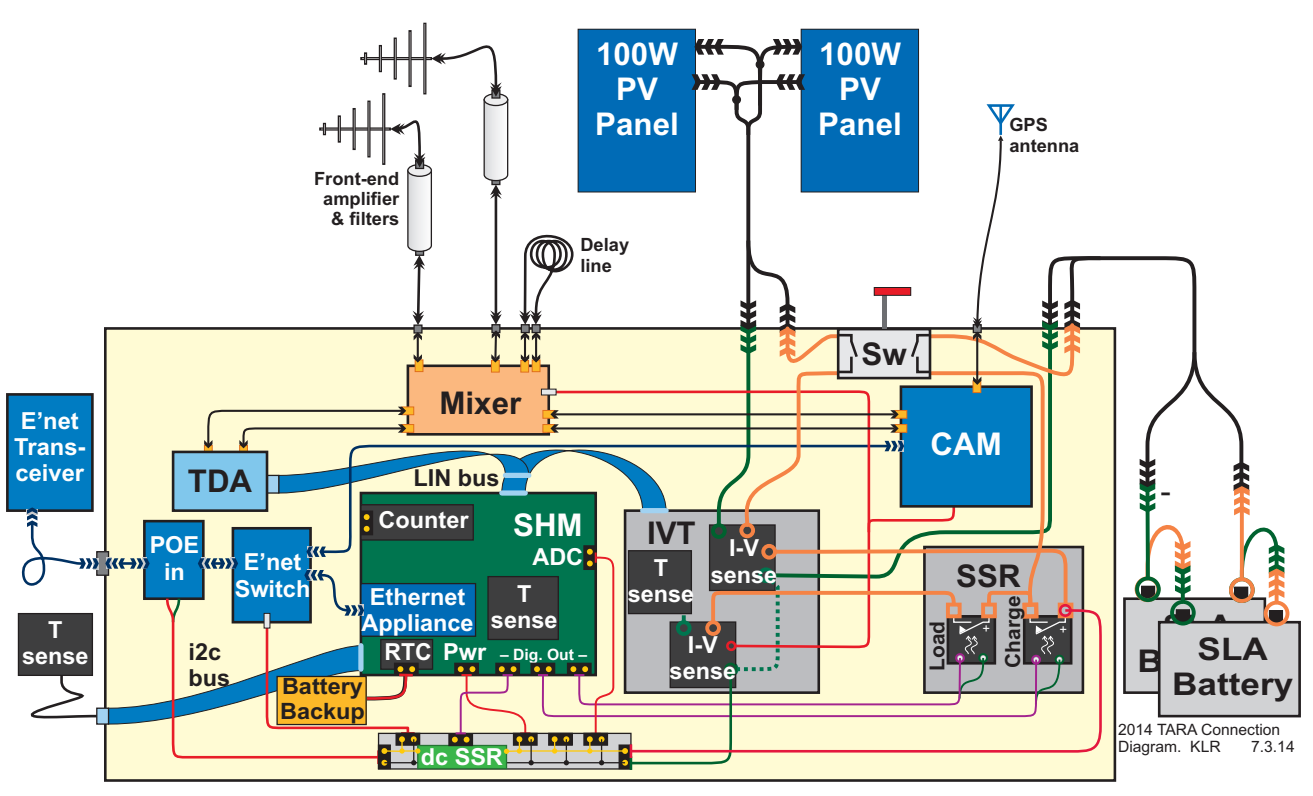

Figure 34: Schematic block diagram of the remote detector electronics. Chirp acquisition module (CAM), power systems, acquisition electronics, and communications blocks are all shown in this figure.

controls, via Solid State Relays (SSRs), the system solar (two 100 Watt photo-voltaic panels) and battery (sealed lead acid) power. The SHM also records antenna data digitized by the TDA receiver on local SD flash memory. The TDA (Transient Detector Apparatus) receiver has two channels with front-end amplifiers, followed by filters and a logarithmic amplifier. Finally, the SHM and
CAM are connected to a $5 \mathrm{GHz}$ Ethernet transceiver via a switch for remote system control and data access.

\subsection{Remote Station Prototype Studies}

To understand the required power budget (Table 5) from the perspective of solar resources in Western Utah, a prototype with system requirements nearly identical 


\begin{tabular}{|l|r|}
\hline Component & Power Consumption(W) \\
\hline \hline Single Board Computer & 5.0 \\
\hline Low Speed 4 Ch. ADC & 0.5 \\
\hline High Speed 1 Ch. ADC & 0.4 \\
\hline FPGA & 3.0 \\
\hline RMS Counter & 2.0 \\
\hline System Health Monitor & 1.0 \\
\hline 60 dB Amplifier (x2) & 4.0 \\
\hline 25 dB Amplifier (x2) & 0.4 \\
\hline GPS & 0.2 \\
\hline GPS and GPS Antenna & 0.4 \\
\hline Communication Antenna & 3.0 \\
\hline \hline Total & 19.9 \\
\hline
\end{tabular}

Table 5: Estimated power budget for the remote station

to those of the current, full scale remote detector station was deployed at the Telescope Array Fluorescence Detector site at Long Ridge, Utah in the spring of 2013.

The deployed hardware included a system health monitor (SHM) to monitor performance and power provision, four data acquisition channels, a $12 \mathrm{~W}$ dummyload and Ethernet communications. The first prototype remote site was deployed several hundred meters from the LR site. Four detector channels include horizontal and vertical polarizations of the standard TARA receiver LPDA, a spiral (frequency-independent) antenna, and $50 \Omega$ terminator for comparison to system noise. Antennas feed four bulkhead connectors through LMR400 coaxial cable, where the signals are amplified and fed into TDA detectors.

TDA detectors record a hit when voltage rises above a tunable threshold (set to $100 \mathrm{mV}$ ). Independent of the presence of "hits", the trigger rate is reported in software over regular $10 \mathrm{~s}$ intervals. The software controlling the detectors and power management of the station is located in micro-controllers on the System Health Monitor (SHM).

The SHM also supports remote communications, however the prototype station was connected to the FD facilities through $200 \mathrm{~m}$ of CAT6 Ethernet cable, with power-over-Ethernet $(\mathrm{PoE})$ offering ample capability to include system monitoring. Several environmental variables in addition to antenna TDA voltage rates are recorded: solar panel current, voltage, battery voltage, the status of the SSRs (the dummy load), temperature measurements, and support for an anemometer. The prototype remote station recorded solar panel power throughout the summer of 2013, quantifying the amount of solar energy available over time. Figure 35 displays the results. Each day, the station consumed ap-
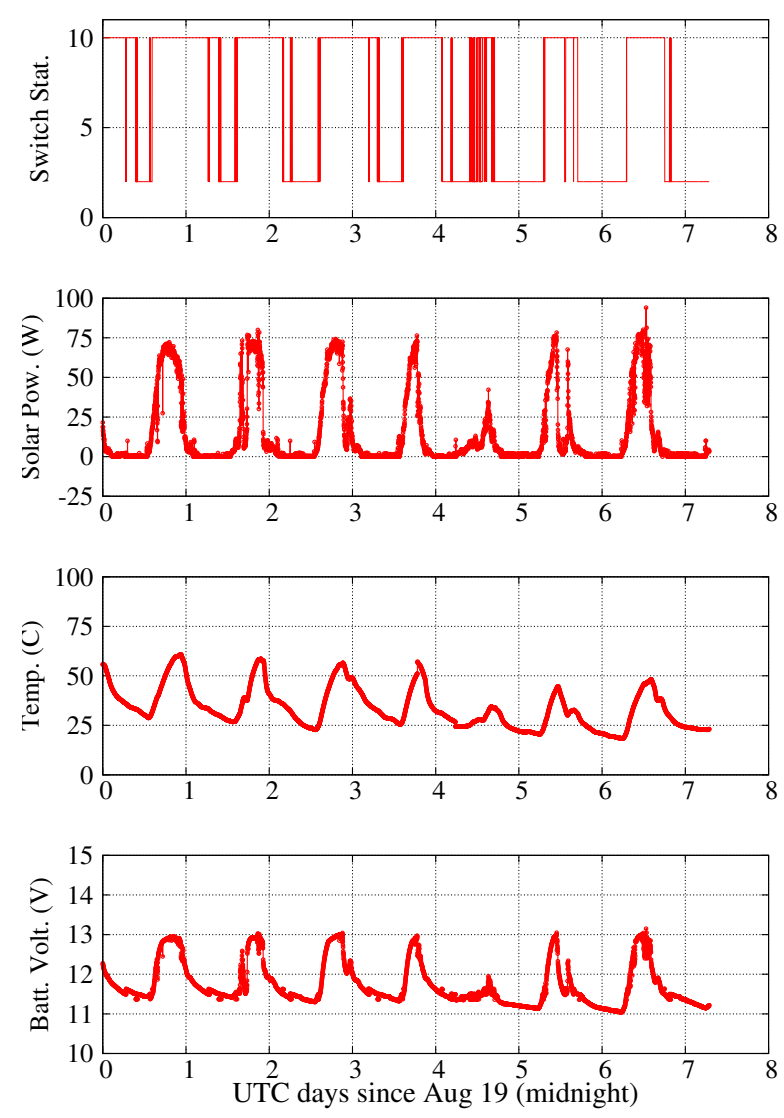

Figure 35: Remote station solar power data for August.

proximately $13 \mathrm{~W}$, or $\approx 26 \mathrm{AHr}$. Data were transmitted via Ethernet and stored locally.

The data show a clear diurnal variation. With the $100 \mathrm{~W}$ rated solar panel oriented toward the sun at 12 p.m. on June 1st, approximately $75 \mathrm{~W}$ peak power delivery was observed. Solar power curves (second from top in Figure 35 have a $3.6 \mathrm{hr}$ full-width halfmaximum, meaning the station collected $21.5 \mathrm{AHr}$ per day. After 40 days the station began to switch off the dummy load at night via the SSRs as the battery became depleted. The $y$-axis of the upper graph is a binary number representing the switch status; 10 (1010 in binary) indicates that both the solar power and dummy load are connected and 2 (0010 in binary) indicates that the dummy load switch has been disconnected by the SHM.

Two improvements to the remote station have been implemented as a result of these prototype data. First, solar photo-voltaic power has been doubled (relative to the prototype) to $200 \mathrm{~W}$ using two panels. The power 
requirement is $20 \mathrm{~W}$ for the current remote station (see Table 5. After accounting for other prototype inefficiencies, two $100 \mathrm{~W}$ panels result in a positive power budget. Second, fine-tuning of SSR shutdown and startup voltages has been implemented in the new remote station to protect the batteries.

The first autonomous remote station was deployed in June 2014, approximately $5 \mathrm{~km}$ NNE of the Long Ridge receiver station. This station and its performance will be described in greater detail in a forthcoming submission to this journal.

\section{Conclusion}

The TARA detector is designed to search for unique cosmic ray radar echoes with very small radar cross sections (RCS). Specifically, the following key characteristics strongly reduce the minimum detectable RCS: high transmitter power $(40 \mathrm{~kW}$, Section 3 ), high-gain transmitter antenna (22.6 dBi, 182 linear, Figure 11. Section 4.2, low noise Radio Frequency (RF) environment consistent with galactic backgrounds (Figure 20, Section 5), innovative triggering scheme that permits detection of signals $7 \mathrm{~dB}$ below the noise (Section 7.4.2), and broadband reciever antenna (12.6 dBi gain, 18.2 linear, Figure 16, Section 5.

Figure 36 shows a calculation of the minimum detectable TARA RCS for a cosmic ray Extensive Air Shower (EAS) located in several positions along a line perpendicular to the transmitter/receiver plane, midway between the transmitter and receiver. The bi-static radar equation (Equation 1, Section 2) permits this simple calculation that assumes a constant power radar echo selftriggered in the DAQ $5 \mathrm{MHz}$ band (Section 7.3.2) with chirp rate in $[-3,-1] \mathrm{MHz} / \mu$ s (Section 7.3). Maximum transmitter/receiver gains are used for each point, given the azimuthal position of the shower core location. Further, the signal is assumed to have constant wavelength and is Doppler-shifted into the DAQ $[60,65] \mathrm{MHz}$ band, for which the $-7 \mathrm{~dB}$ noise floor correction is appropriate, and scattered near the ground (to simplify distance calculation).

The TARA project represents the most ambitious effort to date to detect the radar signature of cosmic ray induced atmospheric ionization. These signals will be characterized by their low power, large Doppler shift (several tens of $\mathrm{MHz}$ ), and short duration $(\sim 10 \mu \mathrm{s})$. TARA combines a high-power transmitter with a stateof-the-art high sampling rate receiver in a low-noise environment in order to maximize the likelihood of cosmic ray echo detection. Importantly, TARA is colocated with the Telescope Array astroparticle observa-

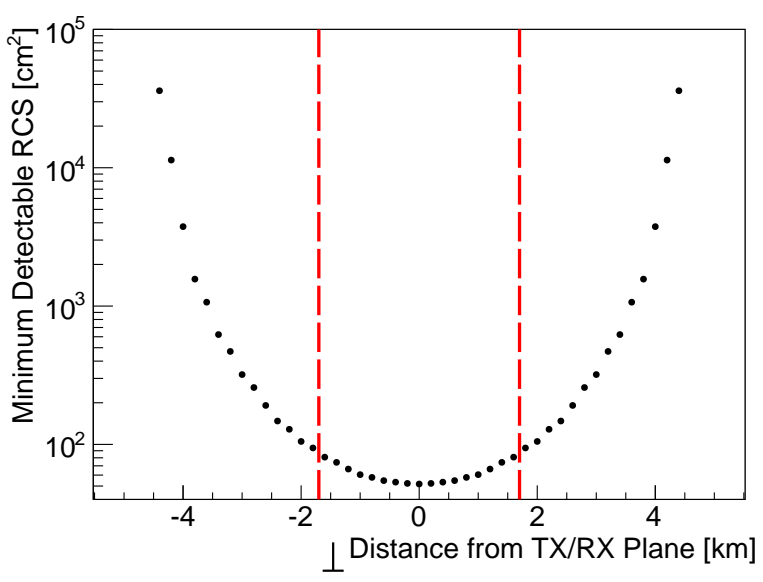

Figure 36: Minimum detectable radar cross section (RCS) as a function of distance perpendicular to the plane connecting the transmitter and receiver. The transmitter antenna main lobe points along this plane. For simplicity, the minimum RCS is calculated from the bi-static radar equation (Equation 1 Section 2 for a cosmic ray air shower midway between transmitter and receiver with maximum transmitter and receiver gains. The $5 \mathrm{MHz}$ FlexRIO passband trigger scheme (Section 7.3.2 was assumed to detect a constant amplitude radar echo with chirp rate in $[-3,-1] \mathrm{MHz} / \mu$ s (Section 7.3 and signalto-noise (SNR) ratio $7 \mathrm{~dB}$ (Section 7.4.2 below background noise (Figure 20 Section 5, the empirical detection performance for the $5 \mathrm{MHz}$ DAQ passband. Futher assumptions are ground-level detection and constant wavelength $\lambda$. Vertical dashed red lines show the $-3 \mathrm{~dB}$ beamwidth of the transmitter antenna.

tory, which will allow for definitive confirmation that any echoes observed are the result of cosmic ray interactions in the atmosphere.

\section{Acknowledgments}

This work is supported by the U.S. National Science Foundation grants NSF/PHY-0969865 and NSF/MRI1126353, by the Vice President for Research of the University of Utah, and by the W.M. Keck Foundation. L. B. acknowledges the support of NSF/REU-1263394. We would also like to acknowledge the generous donation of analog television transmitter equipment by Salt Lake City KUTV Channel 2 and ABC Channel 4, and the cooperation of Telescope Array collaboration.

We would like to specifically thank D. Barr and G. McDonough from Telescope Array for their services.

\section{References}

[1] P. Auger, et al., Extensive Cosmic-Ray Showers, Rev. Mod. Phys. 11 (1939) 288-291.

[2] T. Abu-Zayyad, et al., The Surface Detector Array of the Telescope Array Experiment, Nucl. Instrum. Meth. A 689 (2012) 87 $-97$. 
[3] I. Allekotte, et al., The Surface Detector System of the Pierre Auger Observatory, Nucl. Instrum. Meth. A 586 (2008) 409420.

[4] H. Tokuno, et al., New Air Fluorescence Detectors Employed in the Telescope Array Experiment, Nucl. Instrum. Meth. A 676 (2013) 54-65.

[5] J. Abraham, et al., The Fluorescence Detector of the Pierre Auger Observatory, Nucl. Instrum. Meth. A 620 (2010) 227251.

[6] N.M. Budnev, et. al, The Tunka Experiment: Towards a 1-kmsquare EAS Cherenkov Light Array in the Tunka Valley, Int. J. Mod. Phys. A 20 (2005).

[7] G. Askaryan, Excess Negative Charge of an Electron-Photon Shower And Its Coherent Radio Emission, Sov. J. Exp. Theor. Phys. 14 (1962).

[8] F.D. Kahn and I. Lerche, Radiation from Cosmic Ray Air Showers, P. Roy. Soc. Lond. A 289 (1966).

[9] P.M.S. Blackett, et al., Radio Echoes and Cosmic Ray Showers, Proc. Roy. Soc. A 177 (1941) 183-186.

[10] D. Wahl, et al., The Search for Vertical Extended Air Shower Signals at the Jicamarca Radio Observatory, in: Proceedings of the 30th ICRC, volume 5, 2008, pp. 957-960.

[11] T. Terasawa, et al., Search for Radio Echoes from EAS with the MU Radar, Shigaraki, Japan, in: Proceedings of the 31st ICRC, 2009.

[12] M.F. Bugallo, et. al, MARIACHI: A Multidisciplinary Effort to Bring Science and Engineering to the Classroom, in: IEEE Internation Conference on Acoust., Speech, Signal Process., 2008, pp. 2661-2664.

[13] M.F. Bugallo, et. al, Hands-on Engineering and Science: Discovering Cosmic Rays Using Radar-based Techniques and Mobile Technology, in: IEEE Internation Conference on Acoust., Speech, Signal Process., 2009, pp. 2321-2324.

[14] D.G. Underwood, Large Doppler Shift in RADAR Detection of Ultra-high Energy Eosmic Rays, in: Radar Conference, RADAR '08. IEEE, 2008, pp. 1-5.

[15] M.I. Bakunov, et al., Relativistic Effects in Radar Detection of Ionization Fronts Produced by Ultra-high Energy Cosmic Rays, Astropart. Phys. 33 (2010) 335-340.

[16] N.J. Willis, Bistatic Radar, Scitech Publishing, Raleigh, NC, 1995.

[17] P. Gorham, On the Possibility of Radar Echo Detection of Ultrahigh Energy Cosmic Ray - and Neutrino - induced Extensive Air Showers, Astropart. Phys. 15 (2001).

[18] R.J. Vidmar, On the Use of Atmospheric Pressure Plasmas as Electromagnetic Reflectors and Absorbers, IEEE T. Plasma Sci. 18 (2002).

[19] Y. Itakawa, Effective Collision Frequency of Electrons In Atmospheric Gases, Planet. Space Sci. 19 (1971) 993.

[20] Y. Itakawa, Effective Collision Frequency of Electrons In Gases, Phys. Fluids 16 (1973) 831.

[21] J.W. Crispin and A.L. Maffett, Radar Cross-Section Estimation of Simple Shapes, P. IEEE 53 (1965) 833-848.

[22] T.K. Gaisser and A.M. Hillas, in: Proceedings of the 15th ICRC, volume 8, 1977, p. 353.

[23] H. Takai, et al., Forward Scattering Radar for Ultra High Energy Cosmic Rays, in: Proceedings of the 32nd International Cosmic Ray Conference, 2011.

[24] A. Zech, A Measurement of the Ultra-High Energy Cosmic Ray Flux with the HIRES FADC Detector, Ph.D. thesis, Rutgers University, 2004.

[25] G. Burke, et al., NEC - Numerical Electromagnetics Code for Antennas and Scattering, in: Antennas and Propagation Society International Symposium, volume 17, 1979.

[26] R.U. Abbasi, et al., Indications of Proton-Dominated Cosmic-
Ray Composition Above 1.6 EeV, Phys. Rev. Lett. 104 (2010).

[27] J. Kraus, R. Marhefka, Antennas For All Applications, 3 ed., MgGraw-Hill, New York, NY, 2003.

[28] H.V. Cane, Spectra of the Non-thermal Radio Radiation from the Galactic Polar Regions, Mon. Not. R. Astron. Soc. 189 (1979).

[29] G.A. Dulk, et al., Calibration of Low-frequency Radio Telescopes Using the Galactic Background Radiation, Astron. Astrophys. 365 (2001) 294-300.

[30] M. Othman, et al., On Radar Detection of Chirp Signals with Nondeterministic Parameters in Challenging Noise Background, in: Radar Conference (RADAR), 2013 IEEE, 2013, pp. $1-6$.

[31] J.D. Smith, et al., GPSY2: A Programmable Hardware Module for Precise Absolute Time Event Generation and Measurement, in: Proceedings of the 30th ICRC, volume 5, 2008, pp. 9971000 .

[32] D.Z. Besson, et al., Design, Modeling and Testing of the Askaryan Radio Array South Pole Autonomous Renewable Power Stations, Nucl. Instrum. Meth. A 763 (2014). 